\title{
Control of vein network topology by auxin transport
}

\author{
Carla Verna, Megan G. Sawchuk, Nguyen Manh Linh and Enrico Scarpella*
}

\begin{abstract}
Background: Tissue networks such as the vascular networks of plant and animal organs transport signals and nutrients in most multicellular organisms. The transport function of tissue networks depends on topological features such as the number of networks' components and the components' connectedness; yet what controls tissue network topology is largely unknown, partly because of the difficulties in quantifying the effects of genes on tissue network topology. We address this problem for the vein networks of plant leaves by introducing biologically motivated descriptors of vein network topology; we combine these descriptors with cellular imaging and molecular genetic analysis; and we apply this combination of approaches to leaves of Arabidopsis thaliana that lack function of, overexpress or misexpress combinations of four PIN-FORMED (PIN) genes—PIN1, PIN5, PIN6, and PIN8 — which encode transporters of the plant signal auxin and are known to control vein network geometry.

Results: We find that PIN1 inhibits vein formation and connection, and that PIN6 acts redundantly to PIN1 in these processes; however, the functions of PIN6 in vein formation are nonhomologous to those of PIN1, while the functions of PIN6 in vein connection are homologous to those of PIN1. We further find that PIN8 provides functions redundant and homologous to those of PIN6 in PIN1-dependent inhibition of vein formation, but that PIN8 has no functions in PIN1/PIN6-dependent inhibition of vein connection. Finally, we find that PIN5 promotes vein formation; that all the vein-formation-promoting functions of PIN5 are redundantly inhibited by PIN6 and PIN8; and that these functions of PIN5, PIN6, and PIN8 are independent of PIN1.
\end{abstract}

Conclusions: Our results suggest that PIN-mediated auxin transport controls the formation of veins and their connection into networks.

Keywords: Arabidopsis, Leaf development, Vein network formation, Auxin, PIN genes

\section{Background}

Signals and nutrients are transported within and between the organs of most multicellular organisms by tissue networks. What controls the formation of tissue networks is thus a central question in biology. In animals, many of these networks are stereotyped [1]; by contrast, the vein networks of plant leaves are both reproducible and variable $[2,3]$. Consider, for example, the vein network of an Arabidopsis thaliana leaf [4-10]: lateral veins branch from a single midvein and connect to distal veins to form loops; minor veins branch from midvein and loops, and connect to other veins to form a mesh; and loops and minor veins curve near the leaf margin to lend a scalloped outline to the vein network.

\footnotetext{
* Correspondence: enrico.scarpella@ualberta.ca

Department of Biological Sciences, University of Alberta, Edmonton, Alberta, Canada
}

\section{Biomed Central}

(c) 2015 Verna et al. Open Access This article is distributed under the terms of the Creative Commons Attribution 4.0 International License (http://creativecommons.org/licenses/by/4.0/), which permits unrestricted use, distribution, and reproduction in any medium, provided you give appropriate credit to the original author(s) and the source, provide a link to the Creative Commons license, and indicate if changes were made. The Creative Commons Public Domain Dedication waiver (http://creativecommons.org/publicdomain/zero/1.0/) applies to the data made available in this article, unless otherwise stated.

Geometric features of the vein network such as these are reproducible from leaf to leaf-so much so that they are used as a taxonomic characteristic (e.g., [11]). By contrast, topological features of the vein network are variable $[6,7,10,12-14]$ : the number of veins differs from leaf to leaf, and whether a vein will connect to another vein on both ends or one end will terminate free of contact with other veins is unpredictable; this is always so for minor veins, but even loops can occasionally fail to connect to other veins at one end.

While no evidence is available that associates geometric features of vein networks with the networks' functional traits, abundant evidence exists that associates functional traits of vein networks with the networks' topological features (reviewed in $[15,16]$ ); yet our knowledge of the signals that control vein network topology is 
limited, and most such signals also control vein network geometry (e.g., [17-23]). One of very few exceptions [24-26] is the control of vein network topology by intracellular transport of the plant signal auxin suggested by genetic evidence: leaves of double mutants in the genes encoding the endoplasmic-reticulum (ER)-localized PINFORMED6 (PIN6) and PIN8 auxin transporters of Arabidopsis [27-33] have higher vein-density [33]; the vein density defect of pin6;pin8 leaves is suppressed by mutation of the gene encoding the ER-localized PIN5 auxin transporter [28, 33, 34]; overexpression of PIN6 or PIN8 results in lower vein-density, and overexpression of PIN5 results in the opposite defect [33].

In contrast to the control of vein network topology by intracellular auxin transport, no genetic evidence is available in support of a role for the cell-to-cell transport of auxin in control of vein network topology; yet such a role seems to be suggested by imaging and inhibitor studies. Expression of the PIN1 auxin efflux protein [27, 35] is initiated in broad domains of leaf inner cells that become gradually restricted to files of vascular precursor cells in contact with pre-existing, narrow PIN1 expression domains [33, 36-42]. Within broad expression domains, PIN1 is localized isotropically-or nearly so-to the plasma membrane (PM) of leaf inner cells. As expression of PIN1 becomes gradually restricted to files of vascular precursor cells, PIN1 localization becomes polarized to the side of the PM facing the pre-existing, narrow PIN1 expression domains with which the narrowing domains are in contact. Initially, PIN1 expression domains are in contact with pre-existing domains at one end only, but they can eventually become connected to other PIN1 expression domains at both ends. Inhibitors of cellular auxin efflux delay the restriction of PIN1 expression domains and the polarization of PIN1 localization [38, 39], and induce the formation of more veins $[4,5]$.

The polar localization of PIN1 to the PM of vascular cells-toward pre-existing veins and ultimately the root tip-is thought to determine the polarity of intercellular auxin transport [43]: from the immature shoot-organs, where auxin is produced in large amounts [44, 45], to the roots [46, 47]. By contrast, the directions of ER-PINmediated intracellular auxin-transport are unclear. Available evidence suggests that PIN5 transports auxin from the cytoplasm to the ER lumen [28, 33], and that PIN6 and PIN8 transport it from the ER lumen to the cytoplasm or the nucleus [29-33], the envelope of which is continuous with the ER membrane [48, 49]; alternatively, PIN5, PIN6, and PIN8 could transport in the same direction but have different affinities for different auxins with different developmental functions (e.g., [50]).

Here we asked whether PIN1-mediated intercellular auxin-transport controlled vein network topology and, if so, whether it interacted with the control of vein network topology by ER-PIN-mediated intracellular auxin-transport. To address this question, we introduced descriptors of vein network topology that enable quantification of vein number, connectedness and continuity, and combined these topological descriptors with cellular imaging and molecular genetic analysis to quantify the contribution of PIN1, PIN5, PIN6, and PIN8 to vein network topology. We derived cellular expression and genetic interaction maps of these genes in vein network formation, and suggest that the interaction between PIN1-mediated intercellular auxin-transport and ERPIN-mediated intracellular auxin-transport controls the formation of veins and their connection into networks.

\section{Results and discussion}

\section{Expression of PIN1, PIN5, PIN6, and PIN8 during leaf development}

Veins form sequentially during Arabidopsis leaf development: the formation of the midvein is followed by the formation of the first loops of veins ("first loops"), which in turn is followed by the formation of second loops and minor veins $[4,5,12,13]$ (Fig. 1a-c).

Two distinct auxin-transport pathways have overlapping functions in control of Arabidopsis vein-network geometry [33]. One pathway-mediated by the PMlocalized PIN1 protein-transports auxin intercellularly $[27,35]$; the other pathway-mediated by the ERlocalized PIN5, PIN6, and PIN8 proteins-transports auxin intracellularly [27-34].

Consistent with their role in control of vein network geometry [4, 33, 51, 52], PIN1 (AT1G73590), PIN6 (AT1G77110), and PIN8 (AT5G15100) are expressed in developing veins, although with different dynamics: expression of PIN1 and PIN6 is initiated in broad domains of leaf inner cells, domains that over time become restricted to single files of vascular precursor cells [33, 38-40] (Fig. 1d-i); by contrast, PIN8 expression is restricted from early on to single files of leaf vascular cells [33] (Fig. 1j-1). It remains unclear, however, whether these different dynamics of PIN expression comprise onset of PIN expression at different stages of leaf development.

To address this question, we compared expression of PIN1, PIN6, and PIN8 in first leaves 2, 3 and 4 days after germination (DAG). To visualize PIN expression, we used functional translational fusions (PIN promoter driving expression of the respective PIN:reporter fusion protein) $[33,37,53]$ or transcriptional fusions (PIN promoter driving expression of a reporter protein) [33] (Additional file 1: Table S1); whenever we used transcriptional fusions, their expression matched that of the respective, functional translational fusions [33] (Additional file 2: Figure S1), suggesting that those PIN promoters contain all the regulatory elements required for functional expression of the respective genes. 


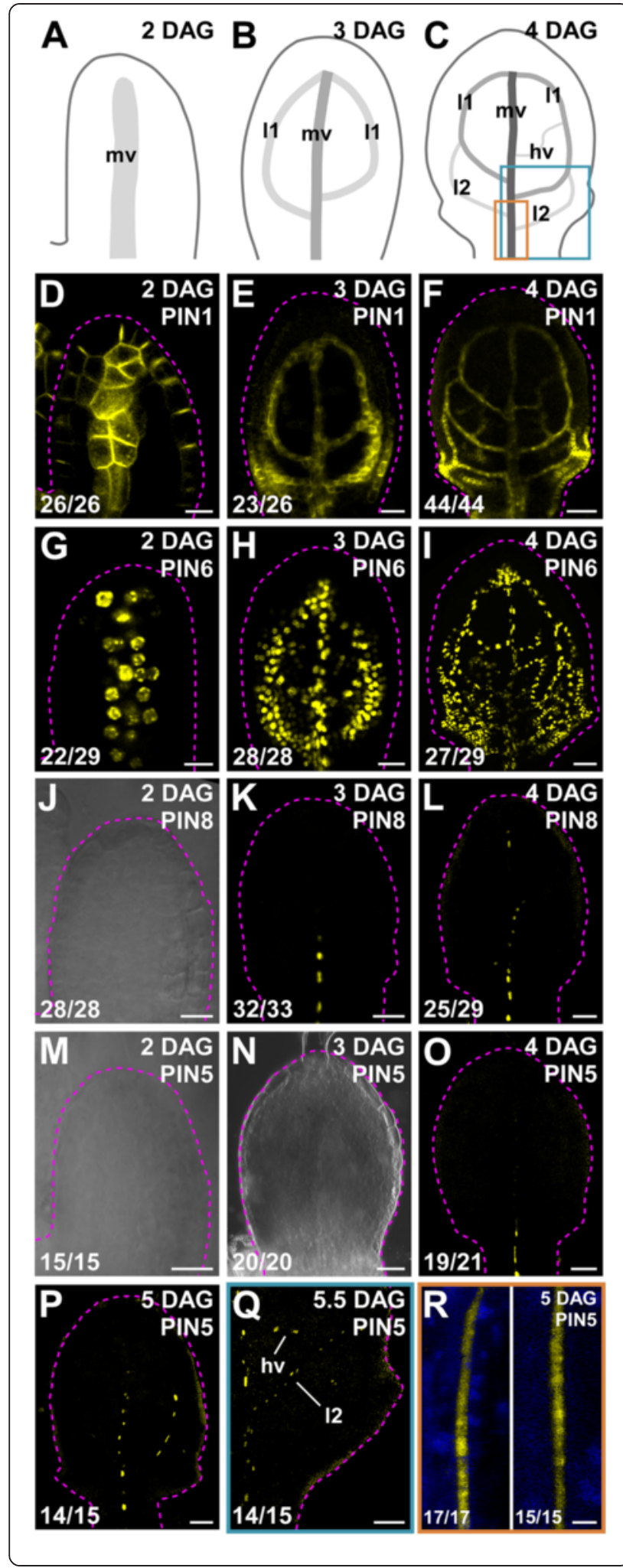

Fig. 1 Expression of PIN1, PIN5, PIN6, and PIN8 of Arabidopsis during first leaf development. a-r. Top right: leaf age in days after germination (DAG) and expression-reported gene (d-r). d-r. Bottom left: reproducibility index. a-c. Midvein, loops and minor veins form sequentially during leaf development $[4,5,12,13]$; increasingly darker gray depicts progression through successive stages of vein development. Boxes in (c) illustrate positions of close-ups in (q) (cyan) and (r) (orange). $\mathbf{d}$-r. Confocal laser scanning microscopy with $(\mathbf{j}, \mathbf{m}, \mathbf{n})$ or without $(\mathbf{d}-\mathbf{i}, \mathbf{k}, \mathbf{l}, \mathbf{o}-\mathbf{r})$ transmitted light; first leaves. Yellow: expression of PIN1::PIN1:GFP (d-f), PIN6::YFPnuc (g-i), PIN8::YFPnuc (j-1), PIN5::YFPnuc ( $\mathbf{m}-\mathbf{q})$, PIN5::PIN5:GFPMGS $(\mathbf{r}$, left) or PIN5::PIN5:GFP ${ }^{\mathrm{AG}}(\mathbf{r}$, right). Blue: autofluorescence $(\mathbf{r})$. Dashed magenta line delineates leaf primordium outline. hv, minor veins; 11, first loop; 12,

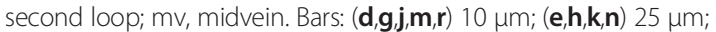
(fi,i,l,o-q) $50 \mu \mathrm{m}$

While expression of a PIN1::PIN1:GFP translational fusion (PIN1 promoter driving expression of PIN1:GFP fusion protein) and of a PIN6::YFPnuc transcriptional fusion (PIN6 promoter driving expression of a nuclear yellow fluorescent protein) was already visible 2 DAG (Fig. 1d, g), expression of PIN8::YFPnuc was first detected 3 DAG (Fig. 1j, k), suggesting that PIN8 expression is initiated after the onset of expression of both PIN1 and PIN6.

PIN5 (AT5G16530) is expressed in veins of mature leaves $[28,54]$, but its expression during leaf development is unknown. Transcriptional and translational fusions of PIN5 are expressed in similar domains [28, 34, 54], suggesting that the PIN5 promoter contains all the regulatory elements required for PIN5 expression. Thus, to visualize PIN5 expression during leaf development, we imaged PIN5::YFPnuc expression in first leaves 2, 3, 4, 5 and 5.5 DAG.

Expression of PIN5::YFPnuc was first detected in the midvein of 4-DAG leaves (Fig. 1m-o); at 5 DAG, PIN5::YFPnuc was additionally expressed in first loops (Fig. 1p), and at 5.5 DAG PIN5::YFPnuc was additionally expressed in second loops and minor veins (Fig. 1q). Thus our results suggest that PIN5 expression is initiated after PIN8 expression (Fig. 1k, n, o) and that, as PIN8, PIN5 is expressed from early on in single files of leaf vascular cells (Fig. 1k, l, o-q).

Expression of PIN5 in single files of leaf vascular cells-suggested by PIN5::YFPnuc expression-was supported by expression of two functional (Additional file 1: Table S1) [34] PIN5::PIN5:GFP translational fusions (Fig. 1r).

\section{Expression of PIN1, PIN5, PIN6, and PIN8 in leaf} vascular cells

Because PIN1, PIN5, PIN6, and PIN8 are all expressed in developing veins (Fig. 1), we asked whether these genes were expressed in the same vascular cells. To address this question, we imaged pairwise combinations of 
fluorescent reporters of PIN1, PIN5, PIN6, and PIN8 in midvein cells of 4-DAG first leaves-where these genes are expressed (Fig. 1f, i, l, o)-and quantified reporter coexpression.

In none of the 20 analyzed leaves coexpressing PIN5::YFPnuc and PIN6::CFPnuc (PIN6 promoter driving expression of a nuclear cyan fluorescent protein) were cells expressing PIN5::YFPnuc ever on the same plane as cells expressing PIN6::CFPnuc: cells expressing PIN5::YFPnuc were located ventrally, while cells expressing PIN6::CFPnuc were located dorsally (Fig. 2a-c). Likewise, in none of the 20 analyzed leaves coexpressing PIN8::YFPnuc and PIN6::CFPnuc were cells expressing
PIN8::YFPnuc ever on the same plane as cells expressing PIN6::CFPnuc: cells expressing PIN8::YFPnuc were located ventrally, while cells expressing PIN6::CFPnuc were located dorsally (Fig. 2d-f). And although cells expressing PIN5::YFPnuc or PIN8::PIN8:GFP ${ }^{\text {MGS }}$ were both on the same ventral plane (Fig. 2g-i), only fewer than $3 \%$ of the cells expressing either reporter expressed both (Fig. 2s).

Approximately $95 \%$ of PIN5::YFPnuc-expressing cells expressed PIN1::PIN1:GFP, but only $\sim 25 \%$ of the PIN1::PIN1:GFP-expressing cells that were on the same ventral plane as cells expressing PIN5::YFPnuc expressed this reporter (Fig. 2j-l, s). Likewise, $\sim 90 \%$ of
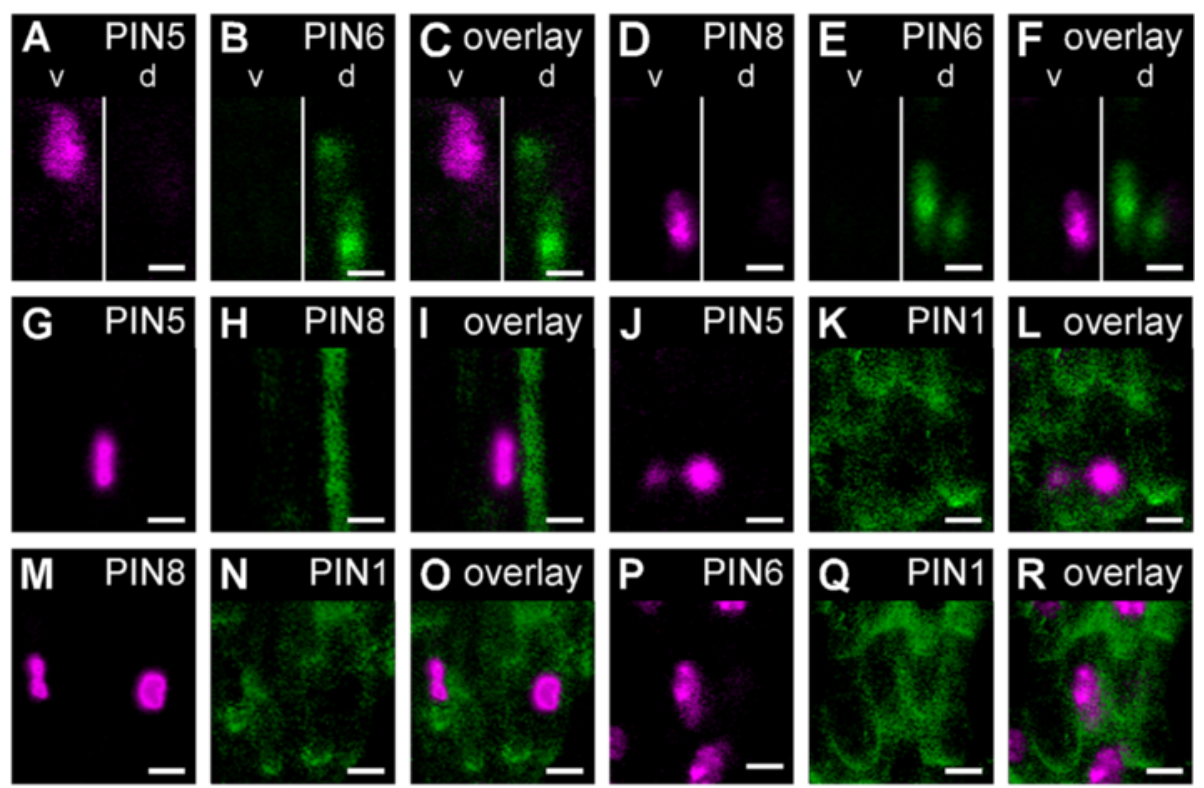

S
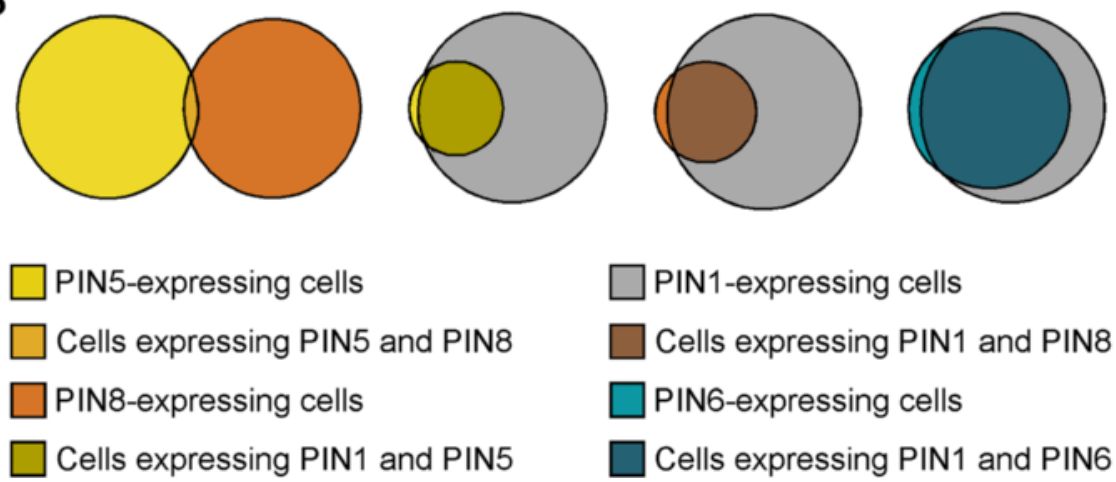

$\square$ PIN1-expressing cells

$\square$ Cells expressing PIN1 and PIN8

]PIN6-expressing cells

\section{Cells expressing PIN1 and PIN6}

Fig. 2 Expression of PIN1, PIN5, PIN6, and PIN8 in leaf vascular cells. a-r. Top right: expression-reported gene. Confocal laser scanning microscopy; first leaves. a-r. Expression of PIN5::YFPnuc (a,g,j), PIN6::CFPnuc (b,e), PIN8::YFPnuc (d,m), PIN8::PIN8:GFPMGS (h), PIN1::PIN1:GFP (k,n,q), PIN6::YFPnuc (p), and respective overlays (c,f,i,l,o,r). s. Proportional Venn diagrams of percentage of cells expressing fluorescent reporters in $25-\mu m$ by $25-\mu m$ midvein regions of 4-day-old first leaves (one region per midvein) in different pairwise combinations of reporters. Sample population sizes: PIN5::YFPnuc;PIN8::PIN8:GFPMGS, 40 leaves (41 PIN5::YFPnuc-expressing cells; 39 PIN8::PIN8:GFPMGS-expressing cells); PIN5::YFPnuc;PIN1::PIN1:GFP, 26 leaves (30 PIN5::YFPnuc-expressing cells; 119 PIN1::PIN1:GFP-expressing cells); PIN8::YFPnuc;PIN1::PIN1:GFP, 25 leaves (34 PIN8::YFPnuc-expressing cells; 122 PIN1::PIN1:GFP-expressing cells); PIN6::YFPnuc;PIN1::PIN1:GFP, 31 leaves (127 PIN6::YFPnuc-expressing cells; 174 PIN1::PIN1:GFP-expressing cells). d, dorsal focal plane; v, ventral focal plane. Bars: (a-r) $5 \mu \mathrm{m}$ 
PIN8::YFPnuc-expressing cells expressed PIN1::PIN1:GFP, but only $\sim 25 \%$ of the PIN1::PIN1:GFP-expressing cells that were on the same ventral plane as cells expressing PIN8::YFPnuc expressed this reporter (Fig. 2m-o, s). Finally, consistent with previous observations [33], 95\% of PIN6::YFPnuc-expressing cells expressed PIN1::PIN1:GFP, and $\sim 75 \%$ of the PIN1::PIN1:GFP-expressing cells that were on the same dorsal plane as cells expressing PIN6::YFPnuc expressed this reporter (Fig. 2p-s).

Thus our results suggest that PIN5, PIN6, and PIN8 are expressed in mutually exclusive domains of leaf vascular cells, and that the PIN1 cellular-expression domain overlaps with - but extends beyond-the ER-PIN cellular-expression domain.

Unique and redundant functions of PIN1, PIN5, PIN6, and PIN8 in control of vein network topology

PIN1, PIN5, PIN6, and PIN8 control vein network geometry $[4,33,51,52]$; we asked what their functions are in control of vein network topology.
To characterize vein network topology, we derived (see Methods and Additional file 3: Figure S2 for details) and used three descriptors based on numerical graph invariants: a cardinality index, a continuity index, and a connectivity index.

The cardinality index is a proxy for the number of "veins" (i.e. stretches of vascular elements that contact other stretches of vascular elements at least at one of their two ends) in a network (Fig. 3a).

The continuity index quantifies how close a vein network is to a network with the same number of veins but in which at least one end of each "vein fragment" (i.e. a stretch of vascular elements that are free of contact with other stretches of vascular elements) contacts a vein. The continuity index ranges from 0 -for a network of sole vein fragments-to 1 -for a network without vein fragments (Fig. 3a).

The connectivity index quantifies how close a vein network is to a network with the same number of veins but in which both ends of each vein or vein fragment
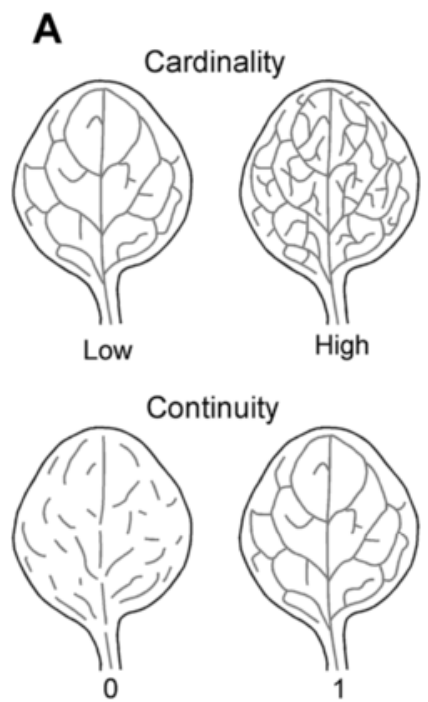

Continuity

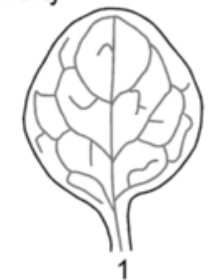

Connectivity
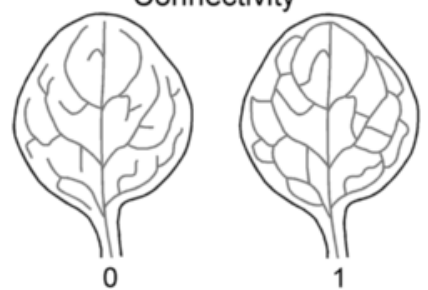

B
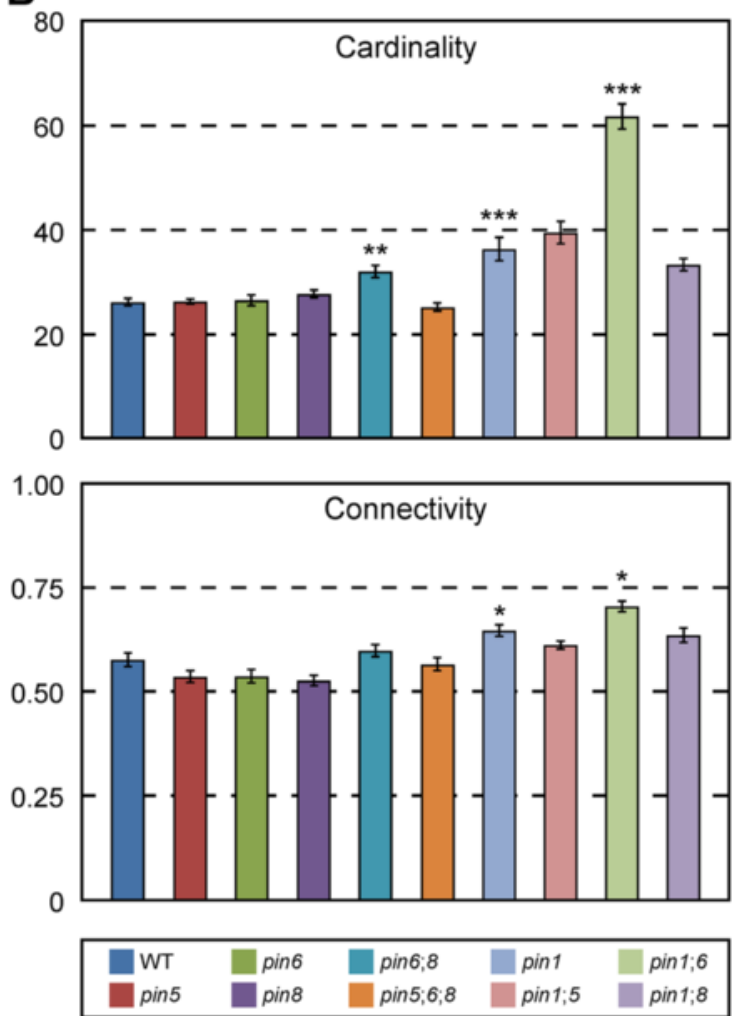

Fig. 3 Functions of PIN1, PIN5, PIN6, and PIN8 in control of vein network topology. a. Schematics of vein networks with low or high cardinality index (top row), minimum - i.e. 0—or maximum —i.e. 1—continuity index (middle row), or minimum—i.e. 0—or maximum—i.e. 1—connectivity index (bottom row). b. First leaves. Indices are expressed as mean \pm SE. Difference between pin6;8 and WT cardinality indices, between pin 1 and WT cardinality indices, between pin 1;6 and pin 1 cardinality indices, between pin 1 and WT connectivity indices, and between pin 1;6 and pin1 connectivity indices was significant at $P<0.05\left({ }^{*}\right), P<0.01(* *)$, or $P<0.001\left(^{* * *}\right)$ by F-test and $t$-test with Bonferroni correction. Sample population sizes: WT, 30; pin5, 30; pin6, 30; pin8, 27; pin6;8, 28; pin5;6;8, 28; pin1, 45; pin1;5, 57; pin1;6, 47; pin1;8, 37 
contact other veins. The connectivity index ranges from 0 -for a network of "open" veins (i.e. veins that contact vein fragments or other veins only at one end) - to 1 -for a network of "closed" veins (i.e. veins that contact vein fragments or other veins at both ends) (Fig. 3a).

Although the number of veins in a leaf is variable and it is unpredictable whether a developing vein will remain open at maturity $[6,7,10,12-14]$, the cardinality and connectivity indices of vein networks in different populations of WT leaves grown in identical conditions were reproducible (Figs. 3, 6, 7 and 8). This observation suggests that while the outcome of vein formation events is unpredictable for single veins, it is predictable-within the limits of statistical variation-for networks of veins. Thus-as for non-stereotyped animal-networks (reviewed in [55])-topology descriptors such as the cardinality and connectivity indices can be compared statistically across genotypes and conditions to identify reproducible patterns and their controls.

The continuity index of vein networks in different populations of WT leaves grown in identical conditions was also reproducible (Additional file 4: Figure S3)-a finding consistent with the stringent requirement for continuity of tissue systems with transport function, such as vein networks, and with the successful use of vein fragmentation as diagnostic criterion for the identification of mutants in genetic screens $[18,56,57]$.

The continuity index of none of the mutants or transgenics in our study was different from that of WT (Additional file 4: Figure S3), suggesting that PIN1, PIN5, PIN6, and PIN8 have no function in control of vein continuity or their functions in this process are redundant.
Consistent with previous observations [33], the vein network topology of pin5, pin6, or pin8 was no different from that of WT (Fig. 3b); by contrast, the cardinality and connectivity indices of pin1 vein networks were higher than those of WT vein networks (Fig. 3b), suggesting that PIN1 inhibits the formation of veins and their connection.

We next asked whether PIN5, PIN6, or PIN8 acted redundantly with PIN1 in inhibition of vein formation and connection. The vein network topology of neither pin1;pin5 (pin1;5 hereafter) nor pin1;8 differed from that of pin1 (Fig. 3b); however, the cardinality and connectivity indices of pin1;6 vein networks were higher than those of pin1 vein networks (Fig. 3b), suggesting that PIN6 acts redundantly with PIN1 in inhibition of vein formation and connection.

Next, we asked whether PIN5 or PIN8 acted redundantly with PIN6 in PIN1-dependent inhibition of vein formation and connection. The vein network topology of pin1;5;6 was no different from that of pin1;6 (Fig. 4), but the cardinality index of pin1;6;8 vein networks was higher than that of pin1;6 vein networks (Fig. 4), suggesting that PIN8 acts redundantly with PIN6 in PIN1-dependent inhibition of vein formation; by contrast, the connectivity index of pin1;6;8 vein networks was no different from that of pin1;6 vein networks (Fig. 4), suggesting that PIN8 has no function redundant to that of PIN6 in PIN1-dependent inhibition of vein connection. Because the vein network topology of neither pin6 nor pin8 differs from that of WT (Fig. 3b), but the cardinality index of pin6;8 vein networks is higher than that of WT (Fig. 3b), PIN6 and PIN8 also

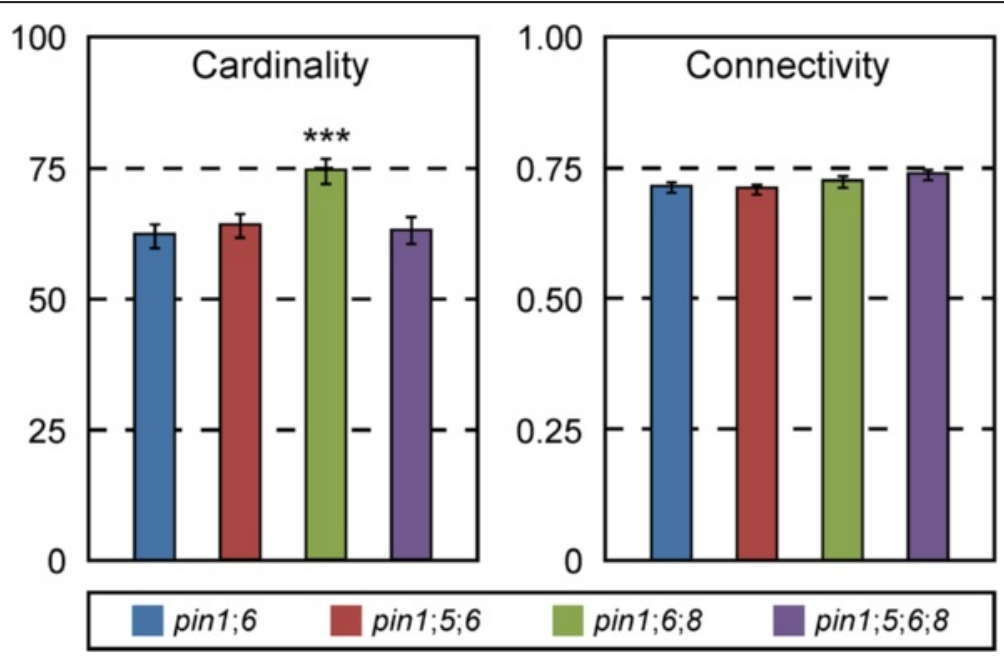

Fig. 4 Functions of PIN5 and PIN8 in PIN1/PIN6-dependent control of vein network topology. First leaves. Indices are expressed as mean \pm SE. Difference between pin 1;6;8 and pin 1;6 cardinality indices was significant at $P<0.001$ (***) by F-test and t-test with Bonferroni correction. Sample population sizes: pin1;6, 103; pin1;5;6, 104; pin1;6;8, 98; pin 1;5;6;8, 109 
have redundant functions in inhibition of vein formation that are independent of PIN1. Thus the enhancement of pin 1;6 cardinality defects by PIN8 could be interpreted as the result of the simultaneous loss of the PIN1-dependent pathway and of the parallel, PIN6/PIN8-dependent, PIN1independent pathway-rather than evidence that PIN8 acts redundantly with PIN6 in PIN1-dependent inhibition of vein formation. However, we do not favor this interpretation because the cardinality defect of pin1;6;8 is much greater than the sum of the cardinality defects of pin1 and pin6;8.

We finally asked whether PIN5 acted redundantly with PIN6 and PIN8 in PIN1-dependent or PIN1-independent inhibition of vein formation. The vein network topology of pin1;5;6;8 was no different from that of pin1;6 (Fig. 4) and that of pin5;6;8 was no different from that of WT (Fig. 3b), suggesting that pin5 suppresses the effects of pin6 and pin8 on PIN1-independent inhibition of vein formation. In agreement with interpretations of similar genetic interactions in other organisms (e.g., [58-60]), the most parsimonious account for our observations is that PIN5 promotes vein formation; that PIN6 and PIN8 redundantly and completely inhibit PIN5-dependent promotion of vein formation; and that these functions of PIN5, PIN6, and PIN8 are independent of PIN1. Further, because expression of PIN5 and PIN8 is initiated at post-formative stages of vein development [33] (Fig. 1), these genes most likely control vein formation indirectly-for example, through feedback on vascular precursor cells located in more-immature parts of the leaf (e.g., [25]; reviewed in [61, 62]). Finally, because PIN5, PIN6, and PIN8 are expressed in non-overlapping sets of vascular cells (Fig. 2), the genetic interaction between these genes-as that between other genes expressed in mutually exclusive domains (e.g., [63-67] and references therein)presumably reflects underlying cell-cell interactions.

\section{Redundant functions of PIN1, PIN6, and PIN8 in control of auxin distribution in developing leaves}

PIN1 inhibits vein formation, and PIN6 acts redundantly with PIN1 in inhibition of vein formation and with PIN8 in PIN1-independent inhibition of vein formation (Figs. 3 and 4). We asked whether such redundancy extended to control of auxin distribution in developing leaves, which is known to control vein formation [4, 5, 23, 33, 38, 39, 68]. To address this question, we imaged expression of the auxin reporter DR5rev::YFPnuc [33, 36, 69] in 4-DAG first leaves of WT, pin6;8, pin1, and pin1;6.

As previously reported [22, 33, 38, 68], in WT the DR5 promoter was strongly active in narrow domains that coincide with sites of vein formation (Fig. 5a). Consistent with previous observations [29-33], DR5rev::YFPnuc expression was weaker in pin6;8 than in WT, but domains of DR5rev::YFPnuc expression were equally narrow in pin6;8 and WT (Fig. 5a, b). Levels of DR5rev::YFPnuc expression were lower, and domains of DR5rev::YFPnuc expression were broader, in pin1 than in WT or pin6;8 (Fig. 5a-d); and DR5rev::YFPnuc
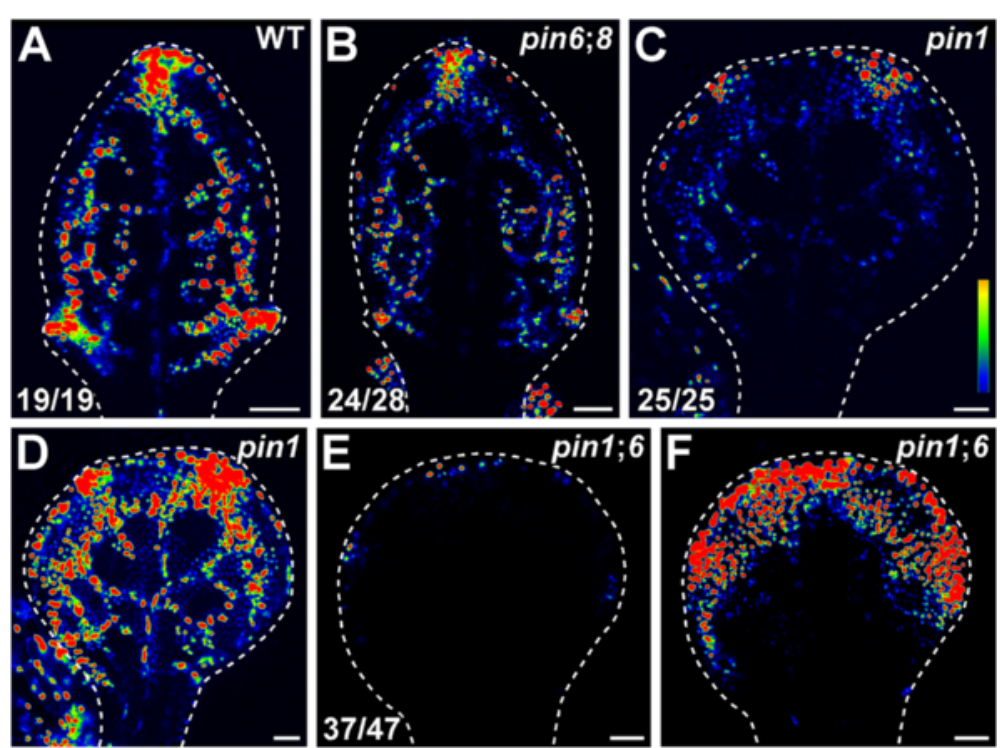

Fig. 5 Expression of DR5rev::YFPnuc in pin developing leaves. a-d. Confocal laser scanning microscopy; first leaves 4 days after germination. Look-up table (ramp in c) visualizes expression levels. Top right: genotype. Bottom left: reproducibility index. Dashed white line delineates leaf primordium outline. Images in $\mathbf{a}, \mathbf{b}, \mathbf{c}$ and $\mathbf{e}$ were taken at identical settings and show increasingly weaker DR5rev::YFPnuc expression in pin6;8, pin 1, and pin 1;6. Images in $\mathbf{a}, \mathbf{d}$ and $\mathbf{f}$ were taken by matching signal intensity to detector's input range ( $4 \%$ saturated pixels), and show increasingly broader DR5rev::YFPnuc expression domains in pin1 and pin1;6. Bars: (a-f) $50 \mu \mathrm{m}$ 
expression levels were even lower, and DR5rev::YFPnuc expression domains even broader, in pin1;6 (Fig. 5c-f).

Thus our results suggest that the redundancy between PIN1, PIN6, and PIN8 that underlies control of vein formation extends to control of auxin distribution in developing leaves (see Conclusions).

\section{Homologous and nonhomologous functions of PIN1 and PIN6 in vein network formation}

PIN6 acts redundantly with PIN1 in control of vein network geometry [33] and topology (Fig. 3b); however, the redundancy between PIN1 and PIN6 is unequal: the geometry and topology of pin6 vein networks are no different from those of WT vein networks but those of pin1 vein networks are, suggesting that PIN1 can provide all-or nearly all-the functions of PIN6 in vein network formation and that, by contrast, PIN6 is unable to provide all the functions of PIN1 in this process. Such unequal redundancy could reflect nonhomologous functions of PIN1 and PIN6 in vein network formation-a possibility consistent with the different localization of PIN1 and PIN6: PIN1 is predominantly localized to the PM [35], while PIN6 is predominantly localized to the ER [33]. On the other hand-at least in other organisms-redundant, homologous functions can be provided by proteins that are localized to different cellular compartments (e.g., [70, 71] and references therein). Further, at least some of the functions of PIN1 in vein network formation depend on PIN1 expression in leaf epidermal cells [51, 72]-leaf epidermal cells that fail, by contrast, to express PIN6 [33] (Fig. 1). Thus the unequal redundancy of PIN1 and PIN6 in vein network formation could alternatively be accounted for by their different expression domains.

To test these possibilities, we used the promoter of the RIBOSOMAL PROTEIN S5A (RPS5A) gene (AT3G11940)-highly active in developing organs, including their epidermal cells [73] - to express PIN1 (RPS5A::PIN1) or PIN6 (RPS5A::PIN6) in the pin1 background, and compared phenotype features of RPS5A::PIN1;pin1 and RPS5A::PIN6;pin1 with those of pin1 and WT.

We first asked whether PIN6 could provide functions in control of vein network geometry homologous to those of PIN1. The geometry of $\sim 15 \%$ of the vein networks of RPS5A::PIN1 and RPS5A::PIN6, and-as previously reported [33] - of nearly $50 \%$ of pin 1 vein networks was abnormal (Fig. 6a-d). RPS5A::PIN1 shifted the spectrum of vein network geometries of pin1 toward the vein network geometry of WT but RPS5A::PIN6 failed to do so (Fig. 6a-d), suggesting that PIN6 is unable to provide functions in control of vein network geometry homologous to those of PIN1.

We next asked whether PIN6 could provide functions in control of vein network topology homologous to

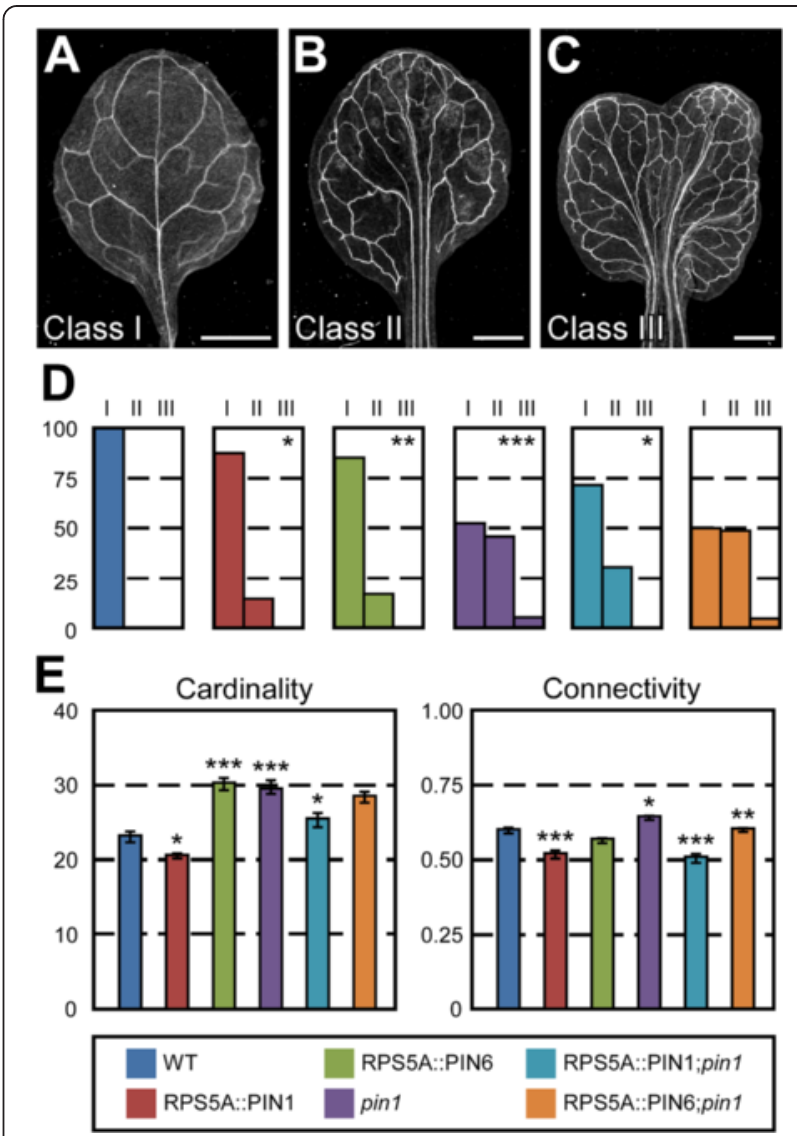

Fig. 6 Functions of PIN1 and PIN6 in vein network formation. a-c. Dark-field illumination of mature first leaves illustrating phenotype classes: unbranched, narrow midvein and scalloped vein-network outline (a); bifurcated midvein and scalloped vein-network outline (b); fused leaves with scalloped vein-network outline (c). d. Percentages of leaves in phenotype classes. Difference between RPS5A::PIN1 and WT, between RPS5A::PIN6 and WT, between pin 1 and WT, and between RPS5A::PIN1; pin1 and pin1 was significant at $P<0.05\left(^{*}\right)$, $P<0.01\left(^{* *}\right)$, or $P<0.001{ }^{(* *)}$ by Kruskal-Wallis and Mann-Whitney test with Bonferroni correction. Sample population sizes: WT, 65; RPS5A:PIN1, 55; RPS5A:PIN6, 58;pin1, 116; RPS5A:PIN1;pin1, 71; RPS5A:PIN6;pin1, 140. e. First leaves. Indices are expressed as mean \pm SE. Difference between RPS5A:PIN1 and WT cardinality indices, between RPS5A::PIN6 and WT cardinality indices, between pin 1 and WT cardinality indices, between RPS5A:PIN1;pin1 and pin1 cardinality indices, between RPS5A:PIN1 and WT connectivity indices, between pin 1 and WT connectivity indices, between RPS5A::PIN1;pin 1 and pin 1 connectivity indices, and between RPS5A:PIN6;pin1 and pin 1 connectivity indices was significant at $P<0.05(*), P<0.01(* *)$, or

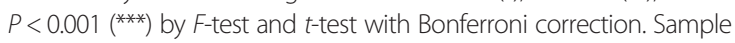
population sizes as in (d). Bars: (a-c) $1 \mathrm{~mm}$

those of PIN1. The cardinality and connectivity indices of RPS5A::PIN1 vein networks were lower than those of WT vein networks (Fig. 6e), supporting that PIN1 inhibits vein formation and connection. The cardinality index of RPS5A::PIN6 vein networks was higher than that of WT vein networks (Fig. 6e), suggesting that ectopic expression of PIN6 in the epidermis promotes vein 
formation. As reported above (Fig. 3b), the cardinality and connectivity indices of pin1 vein networks were higher than those of WT vein networks (Fig. 6e). RPS5A::PIN1 shifted the cardinality index of pin1 vein networks toward that of WT vein networks but RPS5A::PIN6 failed to do so (Fig. 6e), suggesting that PIN6 is unable to provide functions in vein formation homologous to those of PIN1. By contrast, both RPS5A::PIN1 and RPS5A::PIN6 shifted the connectivity index of pin1 vein networks toward that of WT vein networks (Fig. 6e), suggesting that PIN6 can provide functions in vein connection homologous to those of PIN1. Interpretations of similar genetic interactions in other organisms (e.g., [74-76]) suggest that the suppression of vein connectedness defects of pin1 by RPS5A::PIN6 can be accounted for by at least two mechanisms. One possibility is that PIN6 acts downstream of PIN1 in the same pathway that controls vein connection; we do not favor this hypothesis, however, because it fails to predict the observed (Figs. 3 and 4) enhancement of vein connectedness defects of pin1 by pin6. Alternatively, vein connection may be unfavored at high auxin levels [77], which would be the result of at least two separate pathways: PIN1-mediated auxin transport toward sites of vein formation [38-41] (Fig. 5) and PIN6-mediated increase in auxin levels within developing vascular cells [31-33] (Fig. 5) (see Conclusions).

In addition to vein network formation, PIN6 acts redundantly with PIN1 in cotyledon patterning, and as in vein network formation, the redundancy between PIN1 and PIN6 in cotyledon patterning is unequal [33]. We thus asked whether PIN6 could provide functions in cotyledon patterning homologous to those of PIN1; our results (Additional file 5: Figure S4) suggest that it cannot.

Finally, RPS5A::PIN1 reverted the pin-shaped, sterile inflorescences of pin1 to WT-looking, fertile inflorescences but RPS5A::PIN6 failed to do so (Additional file 6: Figure S5), suggesting that PIN6 is unable to provide functions in inflorescence development homologous to those of PIN1.

In summary, PIN6 was unable to provide functions homologous to those of PIN1 in control of vein network geometry, vein formation, cotyledon patterning, and inflorescence development. Thus the unequal redundancy between PIN1 and PIN6 in these processes is unlikely to be the result of their different expression and might instead be accounted for by their nonhomologous functions-a conclusion consistent with the opposite effects of PIN1 and PIN6 on intercellular auxin transport [32]. By contrast, PIN6 was able to provide functions in vein connection homologous to those of PIN1, suggesting that PIN6 expression normally limits the ability of PIN6 to compensate for the effects of loss of PIN1 function in vein connection.

\section{Homologous functions of PIN6 and PIN8 in PIN1-dependent vein-network formation}

PIN8 acts redundantly with PIN6 in PIN1-dependent control of vein network geometry [33] and vein formation (Fig. 4); however, the redundancy between PIN6 and PIN8 in PIN1-dependent control of vein network formation is unequal: the geometry and cardinality index of pin $1 ; 8$ vein networks are no different from those of pin1 vein networks, but those of pin1;6 vein networks are; thus PIN6 can provide all the functions of PIN8 in PIN1-dependent control of vein network geometry and vein formation, but PIN8 is unable to provide all the functions of PIN6 in these processes. Further, PIN8 seems to have no function in PIN1/PIN6-dependent vein connection. The unequal functions of PIN6 and PIN8 in vein network formation could be accounted for by the different expression of PIN6 and PIN8 during vein development [33] (Figs. 1 and 2), but it could also reflect nonhomologous functions of PIN6 and PIN8 in this process.

To test these possibilities, we expressed PIN6 or PIN8 by the promoter of the MONOPTEROS $(M P)$ gene (AT1G19850) (MP::PIN6 or MP::PIN8)—highly active in developing veins [33] - in the pin1;6 background, and compared defects of MP::PIN6;pin1;6 and MP::PIN8; pin1;6 with those of pin1;6 and pin1.

We first asked whether PIN8 could provide functions in PIN1-dependent control of vein network geometry homologous to those of PIN6. As previously reported [33], the vein network geometry of MP::PIN6 and MP::PIN8 was no different from that of WT (Fig. 7a-e). By contrast, the geometry of nearly $60 \%$ of pin 1 vein networks was abnormal, and pin6 shifted the spectrum of vein network geometries of pin1 toward more severe phenotype classes [33] (Fig. 7a-e). The spectrum of vein network geometries of MP::PIN6;pin1;6 was no different from that of $\operatorname{pin} 1$ and that of MP::PIN8;pin1;6 was no different from that of MP::PIN6;pin1;6 (Fig. 7a-e), suggesting that PIN8 can provide functions in PIN1dependent control of vein network geometry homologous to those of PIN6.

We next asked whether PIN8 could provide functions in PIN1-dependent control of vein network topology homologous to those of PIN6. Consistent with previous observations [33], MP::PIN6 and MP::PIN8 induced similar defects-as it frequently results from overexpression of genes with homologous functions (e.g., [78-80]): the cardinality and connectivity indices of both MP::PIN6 and MP::PIN8 vein networks were lower than those of WT vein networks (Fig. 7f), supporting that PIN6 inhibits vein formation and connection, and suggesting that PIN8 can inhibit vein connection in addition to vein formation. As reported above (Fig. 3b), the cardinality and connectivity indices of pin 1 vein networks were higher than those of WT vein networks and those of pin1;6 vein networks were 

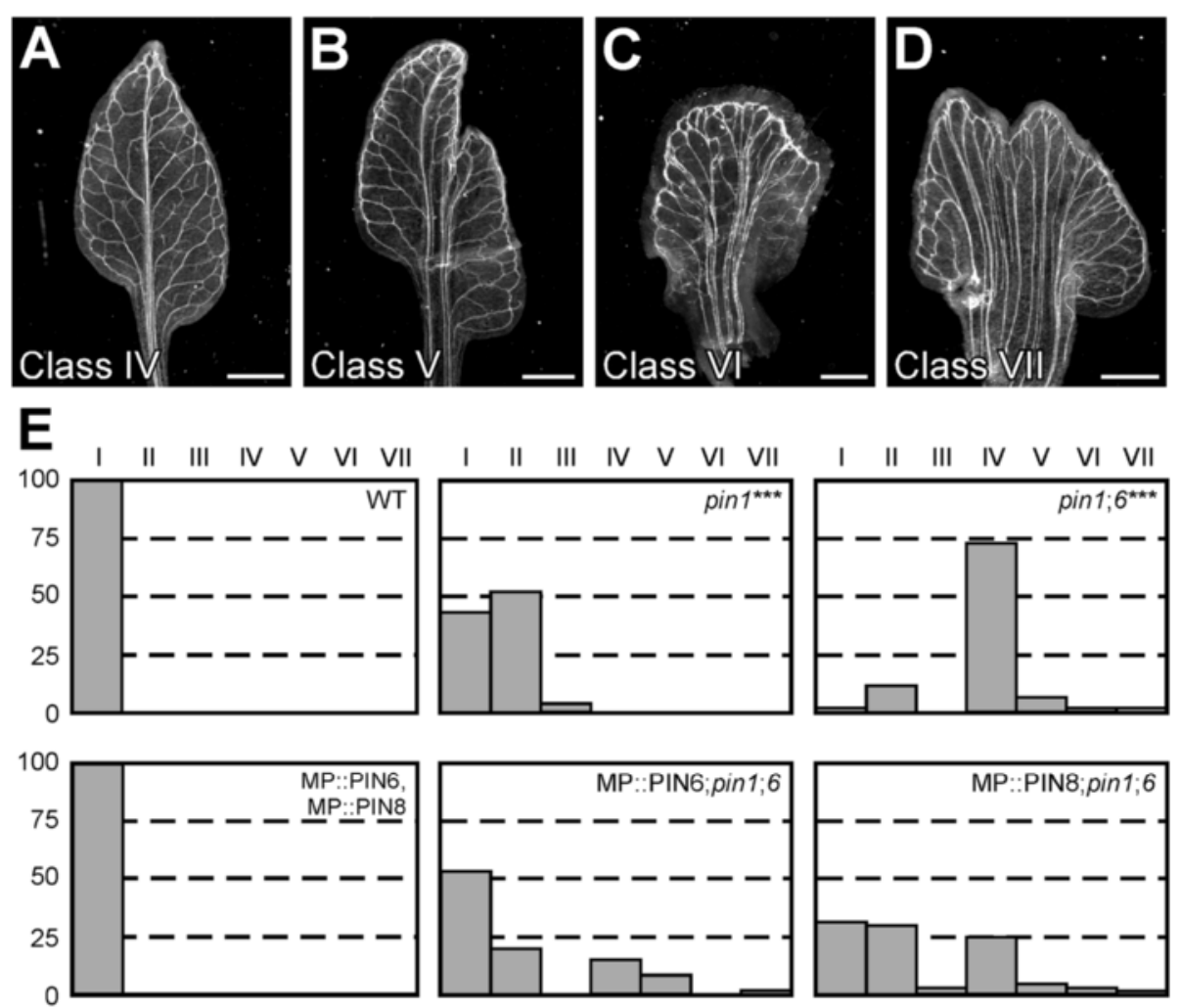

F

Cardinality

Connectivity

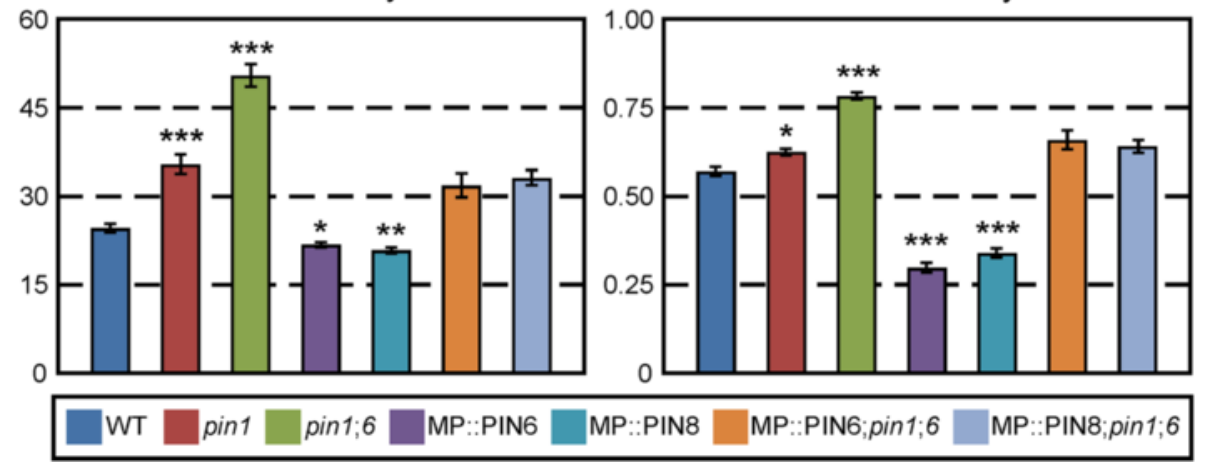

Fig. 7 Functions of PIN6 and PIN8 in PIN1-dependent vein network formation. a-d. Dark-field illumination of mature first leaves illustrating phenotype classes: conspicuous marginal vein (a); fused leaves with conspicuous marginal vein (b); wide midvein (c); fused leaves with wide midvein (d). Phenotype classes I-III as in Fig. 6. e. Percentages of leaves in phenotype classes. Difference between pin 1 and WT, and between pin 1;6 and pin 1 was

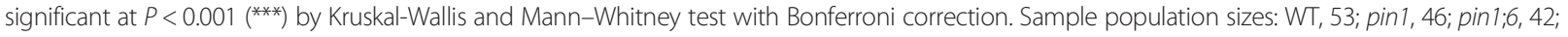
MP.:.PIN6, 54; MP.:.PIN8, 49; MP..:PIN6;pin 1;6, 45; MP.:PIN8;pin 1;6, 60. f. First leaves. Indices are expressed as mean \pm SE. Difference between pin 1 and WT cardinality indices, between pin 1;6 and pin 1 cardinality indices, between MP.:PIN6 and WT cardinality indices, between MP.:PIN8 and WT cardinality indices, between pin 1 and WT connectivity indices, between pin 1;6 and pin 1 connectivity indices, between MP:.:PIN6 and WT connectivity indices, and between MP:.:PIN8 and WT connectivity indices was significant at $P<0.001\left(^{* * *}\right)$ by F-test and $t$-test with Bonferroni correction. Sample population sizes as in (e). Bars: (a,b,d) $1 \mathrm{~mm}$; (c) $0.25 \mathrm{~mm}$

higher than those of pin1 vein networks (Fig. 7f). The vein network topology of MP::PIN6;pin1;6 was no different from that of pin1 and that of MP::PIN8;pin1;6 was no different from that of MP::PIN6;pin1;6 (Fig. 7f), suggesting that PIN8 can provide functions in PIN1-dependent control of vein network topology homologous to those of PIN6.
In addition to PIN1-dependent vein-network formation, PIN8 acts redundantly with PIN6 in PIN1-dependent cotyledon patterning, and as in PIN1-dependent vein network formation, the redundancy between PIN6 and PIN8 in PIN1-dependent cotyledon patterning is unequal [33]. We thus asked whether PIN8 could provide functions in PIN1-dependent cotyledon patterning homologous to 
those of PIN6; our results (Additional file 7: Figure S6) suggest that it can.

In summary, PIN8 was able to provide functions homologous to PIN6 in PIN1-dependent vein network formation and cotyledon patterning. Thus the unequal redundancy between PIN6 and PIN8 is unlikely the result of nonhomologous functions and might instead be accounted for by their different expression. Just as the ER-PIN genes PIN6 and PIN8 redundantly control PIN1dependent vein network formation, the redundancy between the PM-PIN genes PIN1, PIN2, PIN3, PIN4, and PIN7 underlies - to varying extents-many other developmental processes (e.g., [37, 52, 81-84]). In the development of embryos and roots, PM-PIN genes compensate for loss of one another's function by their ectopic expression in the domain of the gene whose function has been lost [82, 84]. For example, in $\operatorname{pin} 7$ embryos PIN4 becomes expressed at earlier stages of development and in the domain in which PIN7 is normally expressed, thereby compensating for loss of PIN7 function [84]. By contrast, in the pin1;6 background PIN8 expression remains restricted to post-formative stages of vein development [33], supporting that PIN8 controls vein network formation by feeding back on vascular precursor cells located in more-immature parts of the leaf.

\section{Functions of PIN5 in PIN6/PIN8-dependent control of vein network topology}

PIN6 has functions in control of vein network topology beyond control of PIN5 function (Fig. 4). We asked whether PIN5 could provide functions in control of vein network topology that are independent of control by PIN6 or PIN8.

To address this question, we used plants expressing PIN5 by the MP promoter (MP::PIN5) because the vein density of MP::PIN5 leaves is higher than that of WT leaves [33]. We reasoned that if PIN5 could provide functions that are independent of control by PIN6 or PIN8, at least some of the effects of MP::PIN5 on vein network topology should persist in the MP::PIN6 or MP::PIN8 backgrounds. By contrast, if all PIN5's functions depended on control by PIN6 or PIN8, the effects of MP::PIN6 or MP::PIN8 on vein network topology should mask those of MP::PIN5.

Consistent with previous observations [33], the cardinality index of MP::PIN5 vein networks was higher than that of WT vein networks (Fig. 8), supporting that PIN5 promotes vein formation. As reported above (Fig. 7), the cardinality and connectivity indices of MP::PIN6 and MP::PIN8 vein networks were lower than those of WT vein networks (Fig. 8). Because the vein network topology of MP::PIN5;MP::PIN6 was no different from that of MP::PIN6 and that of MP::PIN5;MP::PIN8 was no different from that of MP::PIN8 (Fig. 8), we conclude that no function of PIN5 escapes control by PIN6 or PIN8.

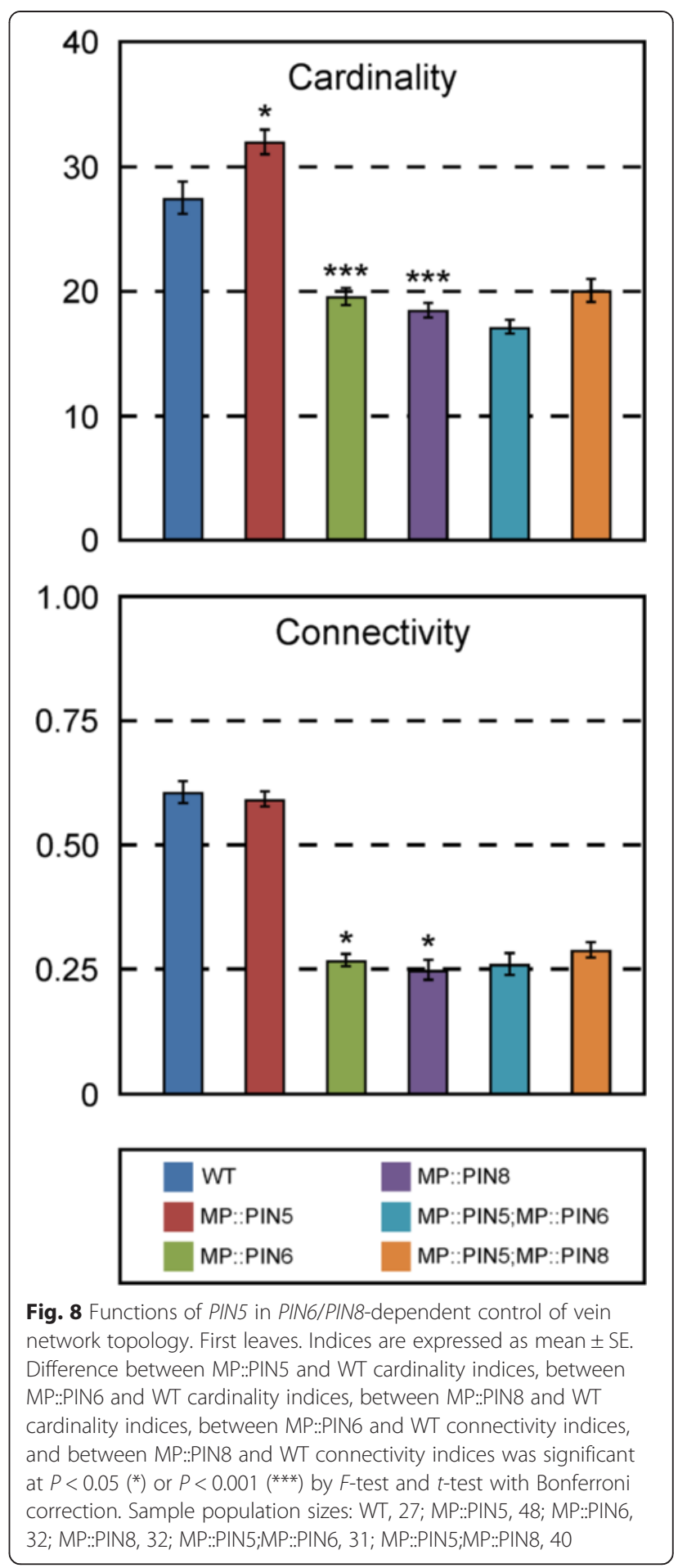

\section{Conclusions}

Vein network formation is redundantly, but nonhomologously, controlled by PIN1-mediated intercellular auxin transport and PIN6/PIN8-mediated intracellular auxin transport (Fig. 9a, b). How to account for such functional overlap? 

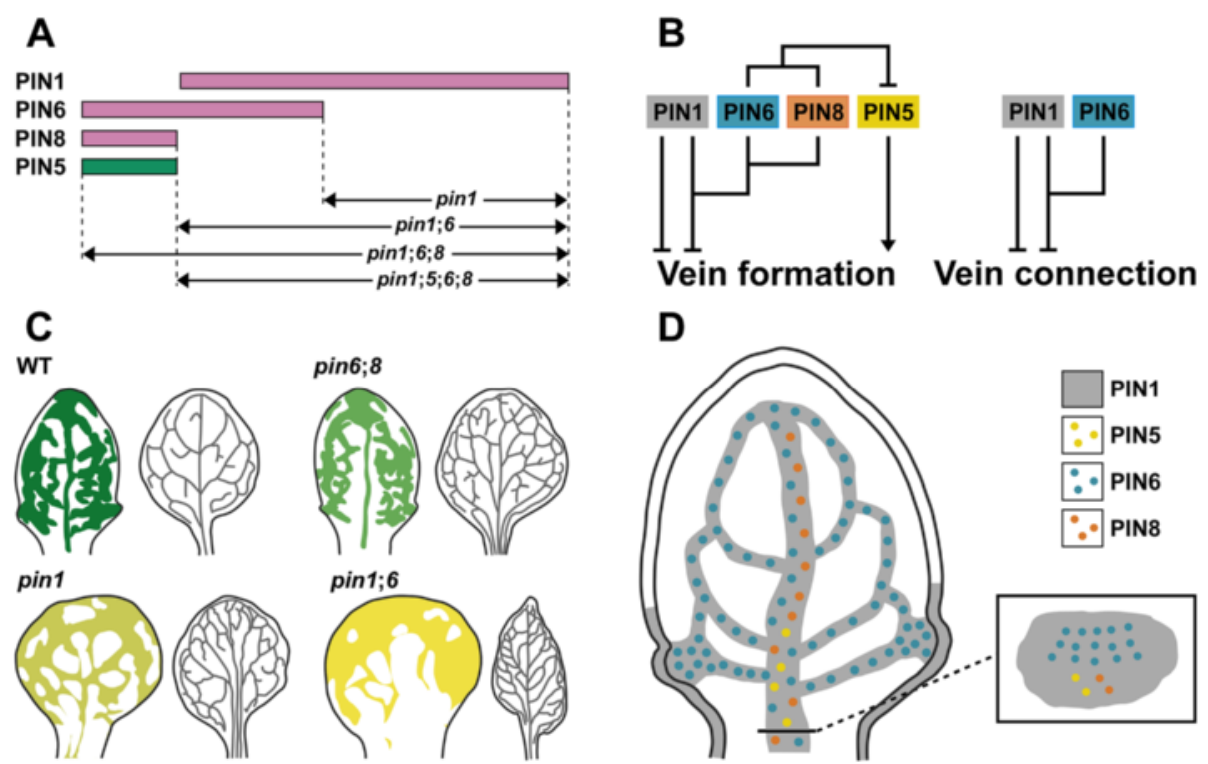

Fig. 9 Summary and interpretations. a. Unique and redundant functions of PIN1, PIN5, PIN6, and PIN8 in vein network formation (magenta, inhibiting functions; green, promoting functions), and derived mutant phenotypes. It is possible that PIN8's functions extend to overlap with PIN6's in PIN1-dependent inhibition of vein network formation. b. Genetic interaction map of PIN1, PIN5, PIN6, and PIN8 in vein network topology. Arrows indicate positive effects; blunt-ended lines indicate negative effects. c. DR5-promoter-activity-derived auxin levels and distribution in developing leaves (left; lower levels are in lighter tints; for simplicity, differences within leaves are ignored), and vein networks in mature leaves (right). $\mathbf{d}$. Cellular expression map of PIN1, PIN5, PIN6, and PIN8 in vein development. See text for details

The "auxin canalization hypothesis" proposes that a positive feedback between auxin movement through a cell and localization of auxin efflux proteins to the site where auxin leaves the cell leads to the selection of cell files from within a field of cells; such cell files would become exposed to inductive levels of auxin, would differentiate into efficient auxin-transport canals-the veins-and would drain auxin from the surrounding areas $[85,86]$. Auxin drainage by developing veins would lower auxin levels in the surrounding areas below those levels which inhibit growth $[87,88]$, growth would resume, and new fields of cells would be generated in which the whole process could be repeated [2].

The predictions of the auxin canalization hypothesis have been rigorously tested and are supported by computer simulation of mathematical models [89-91]; nevertheless, inconsistencies seem to exist between experimental evidence and hypothesis' predictions. For example, the hypothesis appears unable to predict the experimentally observed high levels of auxin in veins $[68,69]$; however, such levels could be, at least in part, the result of PIN1-mediated auxin transport toward sites of vein formation [41] (Figs. 5 and 9d), and of PIN6/ PIN8-mediated increase in auxin levels within developing vascular cells [29-33] (Fig. 5 and 9d). We suggest that because of the lower auxin levels in pin6;8 [29-33] (Fig. 5), auxin would be drained more efficiently in leaves of this background, leaf growth would resume sooner, and veins would form faster-a prediction supported by the faster formation of vein-associated domains of PIN1 expression in pin6;8 [33] - thus leading to the formation of networks of more veins (Fig. 9c). Because of the reduced intercellular auxin-transport in pin1 [92], auxin would accumulate for longer periods in leaves of this background before inducing efficient drainage canals, thereby exposing more cells to inductive levels of auxin-a prediction consistent with the broader domains of DR5rev::YFPnuc expression in pin1 (Fig. 5) - and thus leading to the formation of networks of more veins (Fig. 9c). Because of the additionally lower levels of auxin in pin1;6-suggested by the mimicry of pin1;6 defects by reduction of auxin levels in pin1 [33] and by the lower levels of DR5rev::YFPnuc expression in pin1;6 than in pin1 (Fig. 5) - auxin would accumulate for even longer periods before inducing efficient drainage canals, thereby exposing even more cells to inductive levels of auxin-a prediction consistent with the broader domains of DR5rev::YFPnuc expression in pin1;6 than in pin1 (Fig. 5) - and thus leading to the formation of networks of even more veins (Fig. 9c).

Closed veins form during leaf development from open-vein precursors that become connected with other vein precursors at both ends [12-14, 38-40]. Accounting for the formation of closed veins has long been a challenge for the auxin canalization hypothesis (reviewed in [93-97]). Loss of function of PIN1 and PIN6 leads to 
a network of veins that are more frequently closed, and overexpression of PIN1, PIN6, or PIN8 leads to the opposite defect; thus-consistent with the observation that vein connections form at early stages of tissue development $[12-14,38-40]$ - our results suggest that connection may be favored-or occur exclusively-between vein precursors that have yet to differentiate high auxintransport capacity or high auxin-transport-mediated auxin levels.

\section{Methods}

\section{Definitions, usage and notations}

We define a "vein" as a stretch of vascular elements that contacts another stretch of vascular elements at least at one end (Additional file 3: Figure S2). A stretch of vascular elements that fails to satisfy this requirement is referred to as a "vein fragment" (Additional file 3: Figure S2). We refer to a vein that contacts another vein or a vein fragment only at one end as an "open" vein, and to a vein that contacts vein fragments or other veins at both ends as a "closed" vein (Additional file 3: Figure S2).

We use "vein network geometry" to indicate a set of regularities in the shape and relative position of the veins in a network-e.g., whether the midvein is I- or Y-shaped, and whether the vein network outline is scalloped or smooth-irrespective of the topology of the vein network. We use "vein network topology" to indicate a set of features of a vein network - such as the number of veins and the proportion of closed veins-that are irrespective of the geometry of the vein network.

We use "::" to indicate transcriptional fusions-i.e. fusion of, for example, promoter $A$ to gene $B$-and ":" to indicate translational fusions-i.e. fusion of, for example, gene $A$ to gene $B$. We use semicolons to separate mutations and transgenes in second- and higher-order combinations.

\section{Plants}

Origin and nature of lines, genotyping strategies, and oligonucleotide sequences are in Additional file 1: Table S1, Additional file 8: Table S2, and Additional file 9: Table S3, respectively. Seeds were sterilized and germinated, and plants were grown and transformed as described in [98].

\section{Imaging}

Developing leaves were mounted and imaged as in [33]. Marker-line-specific imaging parameters are in Additional file 10: Table S4 and Additional file 11: Table S5. Mature leaves were fixed in 3:1 ethanol:acetic acid, rehydrated in $70 \%$ ethanol and water, cleared briefly (few seconds to few minutes) in $0.4 \mathrm{M}$ sodium hydroxide, washed in water, and mounted in 1:3:8 water:glycerol:chloral hydrate (Sigma-Aldrich Co. LLC, St. Louis, MO). Mounted leaves were imaged as in [99]. Image brightness and contrast were adjusted by linear stretching of the histogram with ImageJ (National Institutes of Health, Bethesda, MD). Images were cropped with Photoshop (Adobe Systems Inc. San Jose, CA) and assembled into figures with Canvas (ACD Systems International Inc. Victoria, Canada).

\section{Analysis of vein network topology}

We define a "touch point" (TP) as the point where a vein end contacts another vein or a vein fragment, an "end point" (EP) as the point where an open vein terminates free of contact with another vein or a vein fragment, and a "break point" (KP) as each of the two points where a vein fragment terminates free of contact with veins or other vein fragments (Additional file 3: Figure S2). Because it is impractical to determine the TP between a vein that exits a leaf and the vein system of the plant axis, or the KP in proximity of the vein system of the plant axis of a vein fragment that exits a leaf, we define an "exit point" (XP) as the point where a vein or a vein fragment exits the leaf lamina and enters the leaf petiole (Additional file 3: Figure S2), and equate an XP to a TP. The number of TPs, EPs, KPs, and XPs in dark-field images of cleared mature leaves was calculated with the Cell Counter plugin of ImageJ (National Institutes of Health).

A vein network can be understood as an undirected graph in which TPs, EPs, KPs, and XPs are vertices, and veins and vein fragments are edges. Because each vein is incident to two TPs, a TP and an XP, a TP and an EP, or an $\mathrm{XP}$ and an EP, the cardinality index - a measure of the size (i.e. the number of edges) of a graph-is a proxy for the number of veins and is calculated as: $[(\mathrm{TP}+\mathrm{XP}-\mathrm{EP}) / 2]+$ $\mathrm{EP}$, or: $(\mathrm{TP}+\mathrm{XP}+\mathrm{EP}) / 2$.

The continuity index quantifies how close a vein network is to a network with the same number of veins but in which at least one end of each vein fragment contacts a vein, and is thus calculated as the ratio of the cardinality index of the first network to the cardinality index of the second network: $[(\mathrm{TP}+\mathrm{XP}+\mathrm{EP}) / 2] /[(\mathrm{TP}+\mathrm{XP}+\mathrm{EP}+\mathrm{KP}) / 2]$, or: $(\mathrm{TP}+\mathrm{XP}+\mathrm{EP}) /(\mathrm{TP}+\mathrm{XP}+\mathrm{EP}+\mathrm{KP})$.

The connectivity index quantifies how close a vein network is to a network with the same number of veins but in which both ends of each vein or vein fragment contact other veins, and is thus calculated as the ratio of the number of closed veins in the first network to the number of closed veins in the second network (i.e. the cardinality index of the second network): $[(\mathrm{TP}+\mathrm{XP}-\mathrm{EP}) / 2] /[(\mathrm{TP}+$ $\mathrm{XP}+\mathrm{EP}+\mathrm{KP}) / 2]$, or: $(\mathrm{TP}+\mathrm{XP}-\mathrm{EP}) /(\mathrm{TP}+\mathrm{XP}+\mathrm{EP}+\mathrm{KP})$.

\section{Additional files}

Additional file 1: Table S1. Origin and nature of lines [28-31, 33-37, 53, 100]. (DOC $79 \mathrm{~kb}$ )

Additional file 2: Figure S1. Expression of PIN1, PIN6, and PIN8 in Arabidopsis first leaves. (A-H) Confocal laser scanning microscopy; first 
leaves 4 days after germination. Bottom left: reproducibility index and reporter identity. Yellow: expression of PIN1:PIN1:GFP (A), PIN1::PIN1:CFP (B), PIN6::YFPnuc (C), PIN6::CFPnuc (D), PIN6:PIN6:GFPMGS (E), PIN6:PIN6:GFPLLB (F), PIN8::YFPnuc (G), PIN8::PIN8:GFPMGS $(\mathrm{H})$, or PIN8:PIN8:GFPZD (I). Blue: autofluorescence $(\mathrm{A}, \mathrm{F}, \mathrm{H}, \mathrm{I})$. Dashed magenta line delineates leaf primordium outline. Boxes in (E) and (H) illustrate positions of close-ups in (F) and (I),

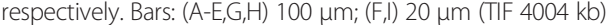

Additional file 3: Figure S2. Analysis of vein network topology. Dark-field illumination of cleared mature first leaf (MP::PIN6) illustrating vein network elements. A "vein fragment" (magenta box) is incident to two "break points" (KPs; magenta dots) - the points where a vein fragment terminates free of contact with veins or other vein fragments. An "open vein" (blue box) is incident to a "touch point" (TP; yellow dot) — a point of contact between a vein and vein fragments or other veins-and an "end point" (EP; blue dot) - the point where an open vein terminates free of contact with another vein or a vein fragment. A "closed vein" (yellow box) is incident to two TPs. A vein or a vein fragment exits the leaf lamina and enters the leaf petiole (green box) by an "exit point" (XP; green dot). See text for details. Bar: $1 \mathrm{~mm}$. (TIF $2596 \mathrm{~kb}$ )

Additional file 4: Figure S3. Functions of PIN1, PIN5, PIN6, and PIN8 in control of vein continuity. First leaves. Indices are expressed as mean \pm SE. Sample population sizes as in Fig. 3B (A), Fig. 4 (B), Fig. 6D (C), Fig. 7E (D), or Fig. 8 (E). (TIF 754 kb)

Additional file 5: Figure S4. Functions of PIN1 and PIN6 in cotyledon patterning. (A-E) Dark-field illumination of 4-day old seedlings illustrating phenotype classes: two separate cotyledons (A); fused cotyledons and separate single cotyledon (B); three separate cotyledons (C); fused cotyledons (D); single cotyledon (E). (F) Percentages of seedlings in phenotype classes. Difference between pin 1 and WT, and between RPS5A:PIN1;pin1 and pin 1 was significant at $P<0.05\left(^{*}\right)$ or $P<0.001$ (***) $^{* *}$ by Kruskal-Wallis and Mann-Whitney test with Bonferroni correction. Sample population sizes: WT, 78; RPS5A:PIN1, 68; RPS5A:PIN6, 32; pin1, 88; RPS5A:PIN1;pin 1, 47; RPS5A:PIN1;pin1, 73. Bars: (A-C) 1 mm; (D,E) 0.5 mm. (TIF $3522 \mathrm{~kb}$ )

Additional file 6: Figure S5. Functions of PIN1 and PIN6 in inflorescence development. (A-F) Four-week-old plants. Top right: genotype. Bottom left: reproducibility index. WT plants normally form fertile flowers (A), while pin 1 plants never do $[92,100]$ (D). Bars: 5 mm. (TIF $1180 \mathrm{~kb}$ )

Additional file 7: Figure S6. Functions of PIN6 and PIN8 in PIN1dependent cotyledon patterning. (A,B) Dark-field illumination of 4-day old seedlings illustrating phenotype classes: partially fused cup-shaped cotyledons, side view; inset: top view (A); completely fused cup-shaped cotyledon, side view; inset: top view (B). Phenotype classes a-e as in Additional file 5: Figure S4. (C) Percentages of seedlings in phenotype classes. Difference between pin 1 and WT, between pin 1;6 and pin1, and between MP::PIN6;pin 1;6 and pin 1;6 was significant at $P<0.01$ (*) $^{*}$ or

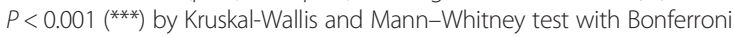
correction. Sample population sizes: WT, 53; pin 1, 52; pin 1;6, 55; MP.:PIN6, 54; MP::PIN8, 49; MP::PIN6;pin 1;6, 47; MP::PIN8;pin 1;6, 62. Bars: (A) 0.5 mm; (B) $0.25 \mathrm{~mm}$. (TIF $1181 \mathrm{~kb}$ )

Additional file 8: Table S2. Genotyping strategies. (DOC $43 \mathrm{~kb}$ ) Additional file 9: Table S3. Oligonucleotide sequences. (DOC 63 kb) Additional file 10: Table S4. Imaging parameters: single-marker lines. (DOC $50 \mathrm{~kb}$ )

Additional file 11: Table S5. Imaging parameters: double-marker lines. (DOC $85 \mathrm{~kb}$ )

\section{Abbreviations}

CFPnuc: Nuclear cyan fluorescent protein; DAG: Days after germination; DR5rev: DIRECT REPEAT5 reverse; ER: Endoplasmic reticulum; GFP: Green fluorescent protein; MP: MONOPTEROS; PIN: PIN-FORMED; PM: Plasma membrane; RPS5A: RIBOSOMAL PROTEIN S5A; YFPnuc: Nuclear yellow fluorescent protein.

\section{Competing interests}

The authors declare that they have no competing interests.

\section{Authors' contributions}

CV, MGS, and ES designed the study; CV, MGS, NML, and ES performed the experiments; CV, MGS, and ES analyzed the data and prepared the manuscript. All authors read and approved the final manuscript.

\section{Acknowledgements}

We thank: Alexander Edgar for help in generating the PIN5::YFPnuc and PIN5:PIN5:GFPMGS lines; Clay Carter, Hyung-Taeg Cho, Pankaj Dhonukshe and Jiří Friml for kindly providing seeds of PIN6::PIN6:GFPRLB , PIN5:PIN5:GFPAG, RPS5A::PIN1, and PIN8::PIN8:GFPZD, respectively; Jason Gardiner for stimulating discussions on analysis of vein network topology. This work was supported by Discovery Grants of the Natural Sciences and Engineering Research Council of Canada (NSERC). CV was supported, in part, by a University of Alberta Doctoral Recruitment Scholarship. MGS was supported, in part, by an NSERC CGS-M Scholarship and an NSERC CGS-D Scholarship. NML was supported by a "322 Project" Scholarship of the Vietnamese Government and a University of Alberta-Vietnam International Education Development Scholarship.

Received: 10 September 2015 Accepted: 28 October 2015

Published online: 11 November 2015

\section{References}

1. Lu P, Werb Z. Patterning mechanisms of branched organs. Science. 2008;322(5907):1506-9

2. Sachs T. The development of vascular networks during leaf development. Curr Top Plant Biochem Physiol. 1989;8:168-83.

3. Berleth T, Mattsson J, Hardtke CS. Vascular continuity and auxin signals. Trends Plant Sci. 2000;5(9):387-93.

4. Mattsson J, Sung ZR, Berleth T. Responses of plant vascular systems to auxin transport inhibition. Development. 1999:126(13):2979-91.

5. Sieburth LE. Auxin is required for leaf vein pattern in Arabidopsis. Plant Physiol. 1999;121(4):1179-90.

6. Candela H, Martinez-Laborda A, Micol JL. Venation pattern formation in Arabidopsis thaliana vegetative leaves. Dev Biol. 1999;205(1):205-16.

7. Kinsman EA, Pyke KA. Bundle sheath cells and cell-specific plastid development in Arabidopsis leaves. Development. 1998;125(10):1815-22.

8. Nelson T, Dengler N. Leaf vascular pattern formation. Plant Cell. 1997;9(7):1121-35.

9. Telfer A, Poethig RS. Leaf development in Arabidopsis. In: Meyerowitz EM, Somerville CR, editors. Arabidopsis. New York: Cold Spring Harbor Press; 1994. p. 379-401.

10. Steynen QJ, Schultz EA. The FORKED genes are essential for distal vein meeting in Arabidopsis. Development. 2003;130(19):4695-708.

11. Klucking EP. Leaf venation patterns. Berlin: J. Cramer; 1995.

12. Scarpella $E$, Francis $P$, Berleth $T$. Stage-specific markers define early steps of procambium development in Arabidopsis leaves and correlate termination of vein formation with mesophyll differentiation. Development. 2004;131(14):3445-55.

13. Kang J, Dengler N. Vein pattern development in adult leaves of Arabidopsis thaliana. Int J Plant Sci. 2004;165(2):231-42.

14. Sawchuk MG, Head P, Donner TJ, Scarpella E. Time-lapse imaging of Arabidopsis leaf development shows dynamic patterns of procambium formation. New Phytol. 2007;176(3):560-71.

15. Sack L, Scoffoni C. Leaf venation: structure, function, development, evolution, ecology and applications in the past, present and future. New Phytol. 2013;198:983-1000.

16. Roth-Nebelsick A, Uhl D, Mosbrugger V, Kerp H. Evolution and function of leaf venation architecture: a review. Ann Bot. 2001;87(5):553-66. doi:10.1006/anbo.2001.1391.

17. Sieburth LE, Muday GK, King EJ, Benton G, Kim S, Metcalf KE, et al. SCARFACE encodes an ARF-GAP that is required for normal auxin efflux and vein patterning in Arabidopsis. Plant Cell. 2006;18(6):1396-411.

18. Koizumi K, Sugiyama M, Fukuda H. A series of novel mutants of Arabidopsis thaliana that are defective in the formation of continuous vascular network: calling the auxin signal flow canalization hypothesis into question. Development. 2000;127(15):3197-204.

19. Petricka JJ, Clay NK, Nelson TM. Vein patterning screens and the defectively organized tributaries mutants in Arabidopsis thaliana. Plant J. 2008:56(2):251-63.

20. Krogan NT, Ckurshumova W, Marcos D, Caragea AE, Berleth T. Deletion of MP/ARF5 domains III and IV reveals a requirement for Aux/IAA regulation in Arabidopsis leaf vascular patterning. New Phytol. 2012;194:391-401. 
21. Garrett JJ, Meents MJ, Blackshaw MT, Blackshaw LC, Hou H, Styranko DM, et al. A novel, semi-dominant allele of MONOPTEROS provides insight into leaf initiation and vein pattern formation. Planta. 2012;236(1):297-312.

22. Donner TJ, Sherr I, Scarpella E. Regulation of preprocambial cell state acquisition by auxin signaling in Arabidopsis leaves. Development. 2009;136(19):3235-46.

23. Cheng $Y$, Dai $X$, Zhao $Y$. Auxin biosynthesis by the YUCCA flavin monooxygenases controls the formation of floral organs and vascular tissues in Arabidopsis. Genes Dev. 2006;20(13):1790-9.

24. Clay NK, Nelson T. Arabidopsis thickvein mutation affects vein thickness and organ vascularization, and resides in a provascular cell-specific spermine synthase involved in vein definition and in polar auxin transport. Plant Physiol. 2005;138(2):767-77.

25. Baima S, Forte V, Possenti M, Penalosa A, Leoni G, Salvi S, et al. Negative feedback regulation of auxin signaling by ATHB8/ACL5-BUD2 transcription module. Mol Plant. 2014;7(6):1006-25.

26. Casson SA, Chilley PM, Topping JF, Evans IM, Souter MA, Lindsey K. The POLARIS gene of Arabidopsis encodes a predicted peptide required for correct root growth and leaf vascular patterning. Plant Cell. 2002;14(8):1705-21.

27. Petrasek J, Mravec J, Bouchard R, Blakeslee JJ, Abas M, Seifertova D, et al. PIN proteins perform a rate-limiting function in cellular auxin efflux. Science. 2006;312(5775):914-8.

28. Mravec J, Skupa P, Bailly A, Hoyerova K, Krecek P, Bielach A, et al. Subcellular homeostasis of phytohormone auxin is mediated by the ER-localized PIN5 transporter. Nature. 2009;459(7250):1136-40.

29. Bosco CD, Dovzhenko A, Liu X, Woerner N, Rensch T, Eismann M, et al. The endoplasmic reticulum localized PIN8 is a pollen specific auxin carrier involved in intracellular auxin homeostasis. Plant J. 2012;71:860-70.

30. Ding Z, Wang B, Moreno I, Duplakova N, Simon S, Carraro N, et al. ER-localized auxin transporter PIN8 regulates auxin homeostasis and male gametophyte development in Arabidopsis. Nat Commun. 2012;3:941.

31. Bender RL, Fekete ML, Klinkenberg PM, Hampton M, Bauer B, Malecha M et al. PIN6 is required for nectary auxin response and short stamen development. Plant J. 2013;74(6):893-904.

32. Cazzonelli Cl, Vanstraelen M, Simon S, Yin K, Carron-Arthur A, Nisar N, et al. Role of the Arabidopsis PIN6 auxin transporter in auxin homeostasis and auxin-mediated development. PLoS One. 2013;8(7), e70069.

33. Sawchuk MG, Edgar A, Scarpella E. Patterning of leaf vein networks by convergent auxin transport pathways. PLoS Genet. 2013;9(2):e1003294.

34. Ganguly A, Park M, Kesawat MS, Cho HT. Functional analysis of the hydrophilic loop in intracellular trafficking of Arabidopsis PIN-FORMED proteins. Plant Cell. 2014;26(4):1570-85.

35. Galweiler L, Guan C, Muller A, Wisman E, Mendgen K, Yephremov A, et al. Regulation of polar auxin transport by AtPIN1 in Arabidopsis vascular tissue. Science. 1998;282(5397):2226-30

36. Heisler MG, Ohno C, Das P, Sieber P, Reddy GV, Long JA, et al. Patterns of auxin transport and gene expression during primordium development revealed by live imaging of the Arabidopsis inflorescence meristem. Curr Biol. 2005;15(21):1899-911.

37. Benkova E, Michniewicz M, Sauer M, Teichmann T, Seifertova D, Jurgens G, et al. Local, efflux-dependent auxin gradients as a common module for plant organ formation. Cell. 2003;115(5):591-602.

38. Scarpella E, Marcos D, Friml J, Berleth T. Control of leaf vascular patterning by polar auxin transport. Genes Dev. 2006;20(8):1015-27.

39. Wenzel $\mathrm{CL}$, Schuetz M, Yu Q, Mattsson J. Dynamics of MONOPTEROS and PIN-FORMED1 expression during leaf vein pattern formation in Arabidopsis thaliana. Plant J. 2007:49(3):387-98.

40. Marcos D, Berleth T. Dynamic auxin transport patterns preceding vein formation revealed by live-imaging of Arabidopsis leaf primordia. Front Plant Sci. 2014;5:235.

41. Bayer EM, Smith RS, Mandel T, Nakayama N, Sauer M, Prusinkiewicz P, et al. Integration of transport-based models for phyllotaxis and midvein formation. Genes Dev. 2009;23(3):373-84.

42. Reinhardt $D$, Pesce ER, Stieger $P$, Mandel T, Baltensperger $K$, Bennett $M$, et al. Regulation of phyllotaxis by polar auxin transport. Nature. 2003;426(6964):255-60

43. Wisniewska J, Xu J, Seifertova D, Brewer PB, Ruzicka K, Blilou I, et al. Polar PIN localization directs auxin flow in plants. Science. 2006;312(5775):883.

44. Thimann KV, Skoog F. On the inhibition of bud development and other functions of growth substance in Vicia faba. Proc R Soc Lond B Biol Sci. 1934;114(789):317-39. doi:10.1098/rspb.1934.0010.
45. Avery Jr GS. Differential distribution of a phytohormone in the developing leaf of Nicotiana, and its relation to polarized growth. Bull Torrey Bot Club. 1935;62(6):313-30

46. Went FW. Wuchsstoff und Wachstum. Recueil des travaux botaniques néerlandais. 1928;25:IV-116.

47. Wangermann E. The pathway of transport of applied indolyl-acetic acid through internode segments. New Phytol. 1974;73(4):623-36.

48. Oda Y, Fukuda H. Dynamics of Arabidopsis SUN proteins during mitosis and their involvement in nuclear shaping. Plant J. 2011;66(4):629-41.

49. Graumann K, Evans DE. Nuclear envelope dynamics during plant cell division suggest common mechanisms between kingdoms. Biochem J. 2011:435(3):661-7.

50. Ludwig-Muller J. Auxin conjugates: their role for plant development and in the evolution of land plants. J Exp Bot. 2012;62(6):1757-73.

51. Bilsborough GD, Runions A, Barkoulas M, Jenkins HW, Hasson A, Galinha C, et al. Model for the regulation of Arabidopsis thaliana leaf margin development. Proc Natl Acad Sci U S A. 2011;108(8):3424-9.

52. Guenot B, Bayer E, Kierzkowski D, Smith RS, Mandel T, Zadnikova P, et al. PIN1independent leaf initiation in Arabidopsis. Plant Physiol. 2012;159(4):1501-10.

53. Gordon SP, Heisler MG, Reddy GV, Ohno C, Das P, Meyerowitz EM. Pattern formation during de novo assembly of the Arabidopsis shoot meristem. Development. 2007;134(19):3539-48.

54. She $W$, Lin $W$, Zhu $Y$, Chen $Y$, Jin $W$, Yang $Y$, et al. The gypsy insulator of Drosophila melanogaster, together with its binding protein suppressor of Hairy-wing, facilitate high and precise expression of transgenes in Arabidopsis thaliana. Genetics. 2010;185(4):1141-50.

55. Thompson PM, Ge T, Glahn DC, Jahanshad N, Nichols TE. Genetics of the connectome. Neuroimage. 2013;80:475-88.

56. Carland FM, Berg BL, FitzGerald JN, Jinamornphongs S, Nelson T, Keith B. Genetic regulation of vascular tissue patterning in Arabidopsis. Plant Cell. 1999;11(11):2123-37.

57. Deyholos MK, Cordner G, Beebe D, Sieburth LE. The SCARFACE gene is required for cotyledon and leaf vein patterning. Development. 2000;127(15):3205-13.

58. Aguilera A, Klein HL. Genetic and molecular analysis of recombination events in Saccharomyces cerevisiae occurring in the presence of the hyper-recombination mutation hpr1. Genetics. 1989;122(3):503-17.

59. Fan HY, Klein HL. Characterization of mutations that suppress the temperature-sensitive growth of the hpr1 delta mutant of Saccharomyces cerevisiae. Genetics. 1994;137(4):945-56.

60. Hudson JD, Feilotter H, Young PG. stf1: non-wee mutations epistatic to cdc25 in the fission yeast Schizosaccharomyces pombe. Genetics. 1990;126(2):309-15.

61. Hsu YC, Fuchs E. A family business: stem cell progeny join the niche to regulate homeostasis. Nat Rev Mol Cell Biol. 2012;13(2):103-14.

62. Hsu YC, Li L, Fuchs E. Emerging interactions between skin stem cells and their niches. Nat Med. 2014;20(8):847-56.

63. Stein KK, Nesmith JE, Ross BD, Golden A. Functional redundancy of paralogs of an anaphase promoting complex/cyclosome subunit in Caenorhabditis elegans meiosis. Genetics. 2010;186(4):1285-93.

64. Hirakawa $Y$, Shinohara $H$, Kondo $Y$, Inoue A, Nakanomyo I, Ogawa M, et al. Non-cell-autonomous control of vascular stem cell fate by a CLE peptide/ receptor system. Proc Natl Acad Sci U S A. 2008;105(39):15208-13.

65. Etchells JP, Turner SR. The PXY-CLE41 receptor ligand pair defines a multifunctional pathway that controls the rate and orientation of vascular cell division. Development. 2008;137(5):767-74.

66. Briscoe J, Pierani A, Jessell TM, Ericson J. A homeodomain protein code specifies progenitor cell identity and neuronal fate in the ventral neural tube. Cell. 2000;101(4):435-45.

67. Sims D, Duchek P, Baum B. PDGFNEGF signaling controls cell size in Drosophila. Genome Biol. 2009;10(2):R20

68. Mattsson J, Ckurshumova W, Berleth T. Auxin signaling in Arabidopsis leaf vascular development. Plant Physiol. 2003;131(3):1327-39.

69. Brunoud G, Wells DM, Oliva M, Larrieu A, Mirabet V, Burrow AH, et al. A nove sensor to map auxin response and distribution at high spatio-temporal resolution. Nature. 2012;482(7383):103-6.

70. Kouranti I, McLean JR, Feoktistova A, Liang P, Johnson AE, Roberts-Galbraith RH et al. A global census of fission yeast deubiquitinating enzyme localization and interaction networks reveals distinct compartmentalization profiles and overlapping functions in endocytosis and polarity. PLoS Biol. 2010;8(9). doi:10.1371/journal.pbio.1000471 
71. Jin Z, Homola E, Tiong S, Campbell SD. Drosophila myt1 is the major cdk1 inhibitory kinase for wing imaginal disc development. Genetics. 2008;180(4):2123-33.

72. Kierzkowski D, Lenhard M, Smith R, Kuhlemeier C. Interaction between meristem tissue layers controls phyllotaxis. Dev Cell. 2013;26(6):616-28.

73. Weijers D, Franke-van Dijk M, Vencken RJ, Quint A, Hooykaas P, Offringa R. An Arabidopsis Minute-like phenotype caused by a semi-dominant mutation in a RIBOSOMAL PROTEIN S5 gene. Development. 2001;128(21):4289-99.

74. Maruyama IN, Miller DM, Brenner S. Myosin heavy chain gene amplification as a suppressor mutation in Caenorhabditis elegans. Mol Gen Genet. 1989;219(1-2):113-8.

75. Hodgkin J. Sex determination in the nematode C. elegans: analysis of tra-3 suppressors and characterization of fem genes. Genetics. 1986;114(1):15-52.

76. Han M, Aroian RV, Sternberg PW. The let-60 locus controls the switch between vulval and nonvulval cell fates in Caenorhabditis elegans. Genetics. 1990;126(4):899-913.

77. Sachs T. On determination of pattern of vascular tissues in peas. Ann Bot. 1968;32(128):781-90.

78. Parkhurst SM, Bopp D, Ish-Horowicz D. X:A ratio, the primary sex-determining signal in Drosophila, is transduced by helix-loop-helix proteins. Cell. 1990;63(6):1179-91.

79. Balcells L, Modolell J, Ruiz-Gomez M. A unitary basis for different Hairy-wing mutations of Drosophila melanogaster. Embo J. 1988;7(12):3899-906.

80. Hadwiger JA, Wittenberg C, Richardson HE, de Barros LM, Reed SI. A family of cyclin homologs that control the G1 phase in yeast. Proc Natl Acad Sci U S A. 1989:86(16):6255-9.

81. Fischer $U$, Ikeda $Y$, Ljung $K$, Serralbo $O$, Singh $M$, Heidstra R, et al. Vectorial information for Arabidopsis planar polarity is mediated by combined AUX1, EIN2, and GNOM activity. Curr Biol. 2006;16(21):2143-9.

82. Blilou I, Xu J, Wildwater M, Willemsen V, Paponov I, Friml J, et al. The PIN auxin efflux facilitator network controls growth and patterning in Arabidopsis roots. Nature. 2005;433(7021):39-44.

83. Friml J, Vieten A, Sauer M, Weijers D, Schwarz H, Hamann T, et al. Efflux-dependent auxin gradients establish the apical-basal axis of Arabidopsis. Nature. 2003:426(6963):147-53.

84. Vieten A, Vanneste S, Wisniewska J, Benkova E, Benjamins R, Beeckman T, et al. Functional redundancy of PIN proteins is accompanied by auxin-dependent cross-regulation of PIN expression. Development. 2005;132(20):4521-31.

85. Sachs T. Cell polarity and tissue patterning in plants. Development. 1991;113 Suppl 1:83-93.

86. Sachs T. Integrating cellular and organismic aspects of vascular differentiation. Plant Cell Physiol. 2000;41(6):649-56. doi:10.1093/pcp/41.6.649.

87. Ljung K, Bhalerao RP, Sandberg G. Sites and homeostatic control of auxin biosynthesis in Arabidopsis during vegetative growth. Plant J. 2001;28(4):465-74.

88. Keller CP, Stahlberg R, Barkawi LS, Cohen JD. Long-term inhibition by auxin of leaf blade expansion in bean and Arabidopsis. Plant Physiol. 2004;134(3):1217-26.

89. Mitchison GJ. Model for vein formation in higher-plants. Proc R Soc Lond B Biol Sci. 1980;207(1166):79-109.

90. Mitchison GJ. The polar transport of auxin and vein patterns in plants. Philos Trans R Soc Lond B Biol Sci. 1981;295(1078):461-71.

91. Rolland-Lagan AG, Prusinkiewicz P. Reviewing models of auxin canalization in the context of leaf vein pattern formation in Arabidopsis. Plant J. 2005;44(5):854-65.

92. Okada K, Ueda J, Komaki MK, Bell CJ, Shimura Y. Requirement of the auxin polar transport system in early stages of Arabidopsis floral bud formation. Plant Cell. 1991;3(7):677-84.

93. Jonsson H, Krupinski P. Modeling plant growth and pattern formation. Curr Opin Plant Biol. 2010;13(1):5-11.

94. Sawchuk MG, Scarpella E. Polarity, continuity, and alignment in plant vascular strands. J Integr Plant Biol. 2013;55(9):824-34

95. Scarpella E, Helariutta Y. Vascular pattern formation in plants. Curr Top Dev Biol. 2010;91:221-65.

96. Smith RS, Bayer EM. Auxin transport-feedback models of patterning in plants. Plant Cell Environ. 2009;32(9):1258-71.

97. Prusinkiewicz P, Runions A. Computational models of plant development and form. New Phytol. 2012;193(3):549-69.
98. Sawchuk MG, Donner TJ, Head P, Scarpella E. Unique and overlapping expression patterns among members of photosynthesis-associated nuclear gene families in Arabidopsis. Plant Physiol. 2008;148(4):1908-24.

99. Odat O, Gardiner J, Sawchuk MG, Verna C, Donner TJ, Scarpella E. Characterization of an allelic series in the MONOPTEROS gene of arabidopsis. Genesis. 2014;52(2):127-33. doi:10.1002/dvg.22729.

100. Goto N, Starke M, Kranz AR. Effect of gibberellins on flower development of the pin-formed mutant of Arabidopsis thaliana. Arabidopsis Inf Serv. 1987;23:66-71.

\section{Submit your next manuscript to BioMed Central and take full advantage of:}

- Convenient online submission

- Thorough peer review

- No space constraints or color figure charges

- Immediate publication on acceptance

- Inclusion in PubMed, CAS, Scopus and Google Scholar

- Research which is freely available for redistribution 